\title{
LES MUTATIONS DE LA COOPÉRATION BELGE AU DÉVELOPPEMENT AU PRISME DES ONG
}

\author{
Justine Contor
}

\section{CRISP | «Courrier hebdomadaire du CRISP »}

2017/38 $n^{\circ} 2363$ | pages 5 à 48

ISSN 0008-9664

Article disponible en ligne à l'adresse :

https://www.cairn.info/revue-courrier-hebdomadaire-du-crisp-2017-38-page-5.htm

\section{Pour citer cet article :}

Justine Contor, « Les mutations de la coopération belge au développement au prisme des ONG », Courrier hebdomadaire du CRISP 2017/38 (n²363), p. 5-48.

DOI 10.3917/cris.2363.0005

Distribution électronique Cairn.info pour CRISP.

(C) CRISP. Tous droits réservés pour tous pays.

La reproduction ou représentation de cet article, notamment par photocopie, n'est autorisée que dans les limites des conditions générales d'utilisation du site ou, le cas échéant, des conditions générales de la licence souscrite par votre établissement. Toute autre reproduction ou représentation, en tout ou partie, sous quelque forme et de quelque manière que ce soit, est interdite sauf accord préalable et écrit de l'éditeur, en dehors des cas prévus par la législation en vigueur en France. Il est précisé que son stockage dans une base de données est également interdit. 
Courrier hebdomadaire

$$
n^{\circ} 2363 \cdot 2017
$$

\section{Les mutations de la coopération belge au développement au prisme des ONG}

Justine Contor

(RiSP 


\section{Courrier hebdomadaire}

Rédacteur en chef : Cédric Istasse

Assistante éditoriale : Fanny Giltaire

Le Courrier hebdomadaire est soutenu par l'Administration générale de l'Enseignement et de la Recherche scientifique de la Fédération Wallonie-Bruxelles. Il est également publié avec l'aide financière du Fonds de la recherche scientifique-FNRS.

Une version numérique du Courrier hebdomadaire est disponible en pay per view (au numéro) et en accès gratuit pour les abonnés sur le site portail de CAIRN (http://www.cairn.info).

Le numéro simple : 6,90 euros - le numéro double : 12,40 euros

Abonnement : 235,00 euros

Souscription, commandes et informations :

CRISP - Place Quetelet, 1A - 1210 Bruxelles

Tél : 32 (0)2 2110180 - Fax : 32 (0)2 2197934

http://www.crisp.be - info@crisp.be

IBAN BE51 310027157662 - Swift BBRUBEBB

Éditeur responsable : Jean Faniel - Place Quetelet, 1A - 1210 Bruxelles

Tous droits de traduction, d'adaptation ou de reproduction par tous procédés, y compris la photographie et le microfilm, réservés pour tous pays.

ISSN 00089664 


\section{TABLE DES MATIÈRES}

INTRODUCTION

1. LA COOPÉRATION BELGE AU DÉVELOPPEMENT : UN BREF HISTORIQUE 7

1.1. Les premières décennies 7

1.2. L'instauration du premier cadre réglementaire (1999-2003) 10

1.2.1. Les constats et recommandations de la commission parlementaire $\quad 10$

$\begin{array}{ll}\text { 1.2.2. La réforme Moreels et ses effets } & 13\end{array}$

2. LES ÉVOLUTIONS RÉCENTES DE LA COOPÉRATION AU DÉVELOPPEMENT : VERS UNE MANAGÉRIALISATION ET UNE COMPLEXIFICATION

2.1. Les conceptions de la Banque mondiale et du Fonds monétaire international 16

2.2. Les conceptions de l'Organisation des Nations unies $\quad 17$

2.3. Les conceptions de l'Organisation de coopération et de développement économiques $\quad 18$

2.4. Synthèse : trois points de vue sur la coopération au développement 19

3. LES ACTEURS DE LA COOPÉRATION BELGE : ÉTAT DES LIEUX ET ÉVOLUTIONS 20

3.1. Préalable : les trois piliers de la coopération belge 20

3.2. La coopération multilatérale $\quad 21$

3.3. La coopération bilatérale directe (gouvernementale) 22

3.3.1. La Direction générale du Développement (DGD) 23

3.3.2. L'Agence belge de développement (Enabel) 24

3.3.3. Le Service de l'évaluation spéciale (SES) 26

3.3.4. La Société belge d'investissment pour les pays en développement (BIO-Invest) 28

3.4. La coopération non gouvernementale $\quad 29$

3.4.1. Les ONG de développement 30

3.4.2. Les acteurs hors ONG $\quad 40$

3.4.3. Mise en perspective 43

3.5. Les acteurs périphériques $\quad 43$

3.5.1. La Belgian First Aid and Support Team (B-FAST) 43

3.5.2. Le Fonds belge pour la sécurité alimentaire (FBSA) 44

$\begin{array}{ll}\text { CONCLUSION } & 45\end{array}$ 


\section{INTRODUCTION}

La coopération au développement, domaine généralement peu visible dans l'agenda politique belge, est aujourd'hui au centre d'un vaste mouvement de réforme comportant un aspect managérial inédit. Dans sa note de politique générale de novembre 2015, le ministre en charge de la Coopération au développement au sein du gouvernement fédéral Michel (N-VA/MR/CD\&V/Open VLD), Alexander De Croo (Open VLD), a présenté ses priorités politiques en la matière, dont un nouveau cadre de référence ${ }^{1}$. Selon la vision du ministre, la coopération belge doit s'aligner davantage sur les engagements internationaux pris par la Belgique et recentrer ses actions, mais aussi réformer les dispositifs publics et associatifs qui sont en charge de sa mise en œuvre. Cette volonté fait référence aux Objectifs pour le développement durable, par lesquels l'ensemble des États membres de l'Organisation des Nations unies (ONU) se sont engagés à lutter contre la pauvreté durant la période 2015-2030. Le ministre a proposé de focaliser l'approche de l'aide publique au développement (APD) belge sur les États considérés comme fragiles, en réduisant le nombre de pays partenaires. Enfin, il a évoqué les conclusions d'un « examen par les pairs » mené en 2015 par le Comité d'aide au développement (CAD) - organe de l'Organisation de coopération et de développement économiques (OCDE) -, qui avait pointé un manque de flexibilité persistant sur le terrain et la nécessité d'une collaboration accrue entre les différents canaux de l'aide belge au développement ${ }^{2}$.

Dans ce contexte, le ministre A. De Croo et son administration ont lancé une opération majeure visant à transformer la gestion des moyens d'action de l'APD belge. Cette réforme cible d'abord et avant tout la composante gouvernementale de la coopération au développement : la Coopération technique belge (CTB) a pris le statut d'agence belge de développement (sous le nouveau nom d'Enabel), ce qui devrait renforcer son autonomie financière et opérationnelle vis-à-vis de l'État belge. Par ailleurs, les organisations non gouvernementales (ONG) associées à la mise en œuvre des programmes d'intervention ont dû se soumettre à la mise en place d'une série d'instruments. En particulier, leurs pratiques ont fait l'objet d'une analyse approfondie sur la base d'une dizaine d'indicateurs de performance définis au préalable (《screening»); cet exercice avait notamment pour ambition de réduire le nombre de structures, afin de diminuer la charge de travail de la Direction générale du Développement (DGD) du SPF Affaires étrangères, Commerce extérieur et Coopération au développement. L’instrument principal de cette vaste réforme est une loi datée du 16 juin $2016^{3}$.

Les moyens et l'organisation de l'aide au développement coordonnée par les autorités publiques belges semblent aujourd'hui à un tournant important de leur histoire.

1 Chambre des représentants, Note de politique générale. Développement international. Agenda numérique, $\mathrm{n}^{\circ}$ 1428/5, 6 novembre 2015, p. 3-17. Cf. aussi Chambre des représentants, Note de politique générale. Coopération au développement, $\mathrm{n}^{\circ}$ 588/6, 14 novembre 2014; Chambre des représentants, Note de politique générale. Développement international 2017, n²111/2, 28 octobre 2016; Chambre des représentants, Note de politique générale. Développement international, $\mathrm{n}^{\circ}$ 2708/3, 17 octobre 2017.

2 Organisation de coopération et de développement économiques, «Examens de l'OCDE sur la coopération pour le développement : Belgique, $2015 »$, septembre 2015, www.oecd.org.

Loi du 16 juin 2016 modifiant la loi du 19 mars 2013 relative à la coopération belge au développement, Moniteur belge, 30 juin 2016. 
L'objet du présent Courrier hebdomadaire est donc de dresser un état des lieux de la coopération belge au développement, en pointant ses évolutions, ses enjeux et ses acteurs. Pour ce faire, il couvre essentiellement la période allant de la fin des années 1990 au milieu de l'année 2017.

L'étude est organisée en trois parties. Le premier chapitre revient brièvement sur l'évolution et sur les transformations de la coopération au développement, au plan international et surtout au plan belge, des années 1960 au début des années 2000. Le deuxième chapitre indique comment et combien les évolutions du contexte international intervenues depuis lors ont amené à une managérialisation et à une complexification croissante du domaine de l'aide au développement. Enfin, le troisième chapitre présente les différents acteurs responsables de la coopération belge et leurs évolutions récentes (dont certaines sont bien entendu à mettre en rapport étroit avec les éléments internationaux présentés dans le deuxième chapitre). Dans ce dernier chapitre, une attention toute particulière est réservée à l'analyse des processus de transformation du secteur des ONG belges de développement. En effet, celles-ci sont des actrices incontournables : elles sont présentes et actives depuis de nombreuses décennies au sein de la coopération belge ; elles forment un des trois canaux d'action de la coopération belge; et elles représentent les sociétés civiles belges et du Sud, ce qui leur confère un caractère profondément démocratique. 


\section{LA COOPÉRATION BELGE AU DÉVELOPPEMENT : UN BREF HISTORIQUE}

C’est dans la période 1960-1990 que la coopération belge au développement se construit, se formalise et monte en puissance. En particulier, ce premier chapitre est centré autour de la grande réforme de 1999 (dite réforme Moreels), qui a profondément restructuré la coopération belge à la veille des années 2000, dessinant les lignes de la politique actuelle.

\subsection{LES PREMIÈRES DÉCENNIES}

La coopération belge au développement débute au sortir de la Seconde Guerre mondiale, suite au processus de décolonisation que soutiennent les instances internationales de l'ONU. Sur le plan institutionnel, avec l'indépendance du Congo en juillet 1960, disparait l'administration coloniale, alors que l'aide au développement est confiée au Ministère des Affaires étrangères. En 1962, le gouvernement Lefevre (PSC-CVP/PSB-BSP) démantèle le Ministère des Affaires africaines (qui a succédé au Ministère du Congo belge et du Ruanda-Urundi, mais a échoué à donner satisfaction) ${ }^{4}$ et crée à sa place une nouvelle structure : l'Office de la coopération au développement (OCD). La mission de celui-ci est de recruter et de former des experts et techniciens belges, de gérer les bourses et de mener à bien des projets conclus avec les pays en développement au moyen d'un appui matériel. En juillet 1965, un ministre-secrétaire d'État à la Coopération au développement et au Commerce extérieur (adjoint au ministre des Affaires étrangères) est nommé à l'occasion de la formation du gouvernement Harmel (PSC-CVP/PSB-BSP) : Ernest Adam (PSC). Ce poste disparaît toutefois dès mars 1966, lors de l'entrée en fonction du gouvernement Vanden Boeynants I (PSC-CVP/PLP-PVV). À partir de 1968, le gouvernement belge dispose systématiquement d'un ministre ou d'un secrétaire d'État en charge de la Coopération au développement ${ }^{5}$, ce qui contribue à donner une réelle visibilité à ce domaine.

4 Cf. P. Develtere, A. Michel, Chronique d'un demi-siècle de coopération belge au développement, Bruxelles, SPF Affaires étrangères, Commerce extérieur et Coopération au développement, s.d. [2010], p. 13-19. Lorsqu'il s'agit d'un secrétaire d'État, la tutelle sur la Coopération au développement est exercée par le ministre en charge des Affaires étrangères. Du 23 juin 1995 au $1^{\text {er }}$ juin 1999, dans le gouvernement Dehaene II, c'est toutefois au Premier ministre qu'est adjoint le secrétaire d'État à la Coopération au développement. Cette configuration atypique s'explique par la volonté de Jean-Luc Dehaene (CVP) d'avoir la tutelle sur la Coopération au développement, afin de résoudre certaines dérives dont des acteurs de la coopération belge, notamment non gouvernementale mais pas seulement, s'étaient rendus coupables 
Cette émergence est marquée par la dynamique de décolonisation et se déroule sur fond de fortes tensions internationales entre deux puissances mobilisant, en matière de développement comme dans d'autres domaines, deux visions du monde : les États-Unis (démocratie libérale) et l'URSS (communisme). Sous le titre «Les étapes de la croissance économique : un manifeste anti-communiste", Walt W. Rostow, qui sera conseiller économique des présidents états-uniens démocrates John F. Kennedy et Lyndon B. Johnson, publie en 1960 un manifeste présentant la vision libérale de la décolonisation et de l'aide au développement ${ }^{6}$. Selon cette perspective, l'aide au développement assurera la croissance économique, pour autant que les élites locales adhèrent aux principes de la modernité libérale sur les plans technologique, économique et social. Ce point de vue développementaliste prend appui sur une vision de modernisation qui se déroule à travers cinq phases : primo, la société traditionnelle ne dispose que de fonctions de production limitées ; secundo, dès que les conditions préalables au décollage sont remplies, la société est en transition ; tertio, pendant la phase de décollage, la société dépasse les difficultés qui s'opposent à une croissance régulière; quarto, la marche vers la maturité se caractérise par une longue période de développement soutenu ; quinto, l'âge ultime est celui de la consommation de masse où la production de biens de consommation et les services sont les principaux secteurs de l'économie. D'après W. W. Rostow, les pays traversent tous ces mêmes étapes, de manière linéaire et séquentielle, pour passer du stade $d u$ sous-développement à celui du développement.

Cette idée selon laquelle tous les "pays en voie de développement» doivent tendre vers la modernité des pays industrialisés (cf. infra) influence les politiques de développement au niveau mondial durant toute la décennie 1970 (qui est considérée comme la décennie du développement par l'ONU). À cette époque, l'Assemblée générale des Nations unies adopte une résolution selon laquelle chaque pays économiquement avancé doit accroître progressivement son aide publique au développement (APD) jusqu'à atteindre $0,7 \%$ de sa richesse au cours de la décennie suivante ${ }^{7}$.

En avril 1971, en Belgique, le ministre de la Coopération au développement du gouvernement Eyskens IV (PSC-CVP/PSB-BSP), Raymond Scheyven (PSC), réforme en profondeur l'OCD. Celui-ci devient l'Administration générale de la Coopération au développement (AGCD), intégrée au Ministère des Affaires étrangères (désormais dénommé Ministère des Affaires étrangères, du Commerce extérieur et de la Coopération au développement). Cette réforme maintient cependant une large autonomie administrative de la structure dans la préparation et l'exécution des projets; ainsi, son administrateur général relève directement du ministre en charge de la Coopération au développement et non du Ministère ${ }^{8}$.

Entre les années 1970 et 1980, l'administration belge innove en recourant au cofinancement avec d'autres bailleurs et en encourageant la constitution d'une coopération

au Rwanda dans les années précédant 1994, en jouant un rôle ambigu dans la polarisation ethnique, ce dont le ministre des Affaires étrangères de l'époque n'avait pas pris la mesure.

T.-G. PARK, D. BARJOT, «W.W. Rostow et le discours sur l'économie en Corée du Sud dans les années $1960 »$, Histoire, économie et société, $25^{\mathrm{e}}$ année, nº 2, 2006, p. 281-289.

L. B. PEARSON et al., Partners in Development: Report of the Commission on International Development, Londres/New York, Pall Mall Press/Praeger Publishers, 1969, p. 148-149 ; M. A. ClemENS, T. J. Moss, "Le mythe des $0,7 \%$ : origines et pertinence de la cible fixée pour l'aide internationale au développement », Afrique contemporaine, $\mathrm{n}^{\circ}$ 2019, 2006, p. 173-201.

8 P. Develtere, A. Michel, Chronique d’un demi-siècle de coopération belge au développement, op. cit. 
universitaire. Le nombre de pays bénéficiaires de l'aide belge augmente également, ce qui contribue à rendre plus complexes l'organisation des programmes ainsi que leur suivi. La politique belge de développement se diversifie, mais ce déploiement est organisé sans prendre appui sur un cadre spécifique et approprié, qui lui assurerait une approche plus structurée.

Dans les années 1980, l'administration belge ouvre son offre de coopération à la finance ; elle renforce sa présence dans les organismes internationaux et mène des études prospectives. Mais, l'AGCD est " au bord de l'asphyxie » ${ }^{9}$. Les politiques sont qualifiées d'imprécises et de changeantes, et il leur est reproché de gérer un nombre de projets trop important. Elles sont dénoncées à la fois pour leur ingérence politique dans les pays bénéficiaires et pour le morcellement des responsabilités qu'elles présentent. Ces politiques manquent d'un cadre légal mais aussi de vision à long terme ${ }^{10}$. Dix ans seront nécessaires pour faire aboutir la réforme proposée suite à un rapport demandé par le gouvernement.

Les années 1990 sont difficiles pour la coopération belge. La réforme débute en 1992, mais elle n'a pas le temps de produire ses effets car l'AGCD rencontre de nouvelles difficultés externes : les trois principaux pays dans lesquels la coopération belge est impliquée en Afrique centrale (Congo, Rwanda et Burundi) sont dans la tourmente et le volume de la coopération baisse donc fortement. À cette époque comme aujourd'hui, il n'existe en effet pas de mécanisme de réallocation des moyens non utilisés dans le cas d'une crise politique, d'une guerre, etc.

À cette époque, la Banque mondiale (BM) et le Fonds monétaire international (FMI) interviennent en appliquant les plans d'ajustements structurels (PAS) au niveau des pays en développement ${ }^{11}$. Cette politique est caractérisée par une série de conditions et de contraintes monétaires, budgétaires et structurelles (par exemple : dévaluer la monnaie locale, diminuer le taux des importations, augmenter les recettes fiscales, concentrer les productions « exotiques », diminuer les dépenses publiques, libéraliser les importations et le système bancaire, assouplir les règles de travail, etc.). La plus grande critique adressée aux PAS est qu'ils ont d'abord été pensés pour l'Amérique latine, et ont ensuite été appliqués à d'autres pays sans tenir compte des réalités et des spécificités propres de ces derniers. De plus, ils reposent sur une vision dite libérale du développement, qui met l'accent sur le développement économique et sur le rôle des entreprises privées, et ils demandent aux pays bénéficiaires de l'aide de s'engager à ouvrir leur économie au reste du monde. Finalement, après de nombreuses critiques et des adaptations intermédiaires, les PAS sont abandonnés au profit d'un nouvel outil : les documents stratégiques pour la réduction de la pauvreté (DSRP) ${ }^{12}$.

Ce court retour socio-historique permet de prendre la mesure des nombreuses difficultés structurelles et organisationnelles rencontrées par la coopération belge et internationale au développement entre 1960 et 1990.

\footnotetext{
Ibidem, p. 45.

Ibidem.

P. VINCENT, Institutions économiques internationales : éléments de droit international économique, $2^{\mathrm{e}}$ éd., Bruxelles, Larcier, 2013.

Banque mondiale, Rapport sur le développement dans le monde 2000/2001. Combattre la pauvreté, Washington/Paris, Eska, 2001, http://documents.banquemondiale.org; R. VIVIEN, «L'annulation de la dette du Tiers Monde », Courrier hebdomadaire, CRISP, n² 2046-2047, 2010.
} 


\section{L'INSTAURATION DU PREMIER CADRE RÉGLEMENTAIRE (1999-2003)}

En Belgique, la seconde moitié des années 1990 est marquée par une profonde crise de la coopération au développement. En 1995, le journal flamand De Morgen interpelle l'opinion publique à propos d'une dizaine de projets jugés trop ambitieux, voire mégalomanes, et dont la pertinence pour les populations locales est remise en cause ${ }^{13}$. La Chambre des représentants met sur pied une commission spéciale chargée du suivi des problèmes de l'AGCD ${ }^{14}$. Cette commission spéciale est constituée de députés PS, PRL, PSC, CVP, VLD, SP et Vlaams Blok ; son président est Dirk Vandermaelen (SP). La méthodologie utilisée est la suivante: étude préalable, examen de documents spécifiques, collaboration avec l'exécutif par l'entremise du secrétaire d'État à la Coopération au développement du gouvernement Dehaene II (CVP/PS/SP/PSC), Réginald Moreels (CVP), et réalisation d'un grand nombre d'auditions (ministres, fonctionnaires, hauts fonctionnaires, représentants d'ONG, chercheurs académiques). Pour l'analyse des modalités de mise en œuvre de la politique, la commission spéciale s'intéresse spécifiquement à un échantillon de trois ONG tirées au hasard: SOS Faim, Médecins sans frontières (MSF) ${ }^{15}$ et Coopibo. Les dossiers de celles-ci sont pris à titre d'exemples pour mener un audit et ainsi analyser, au siège des ONG concernées et sur la base de documents écrits, une série d'éléments en grande partie relatifs à la gestion comptable.

\subsubsection{Les constats et recommandations de la commission parlementaire}

En juillet 1997, la commission spéciale remet un rapport très critique déclarant que la coopération belge au développement est en crise ${ }^{16}$. Ce document recueille l'assentiment de tous les partis, en ce compris ceux de l'opposition. Seul le Vlaams Blok critique l'idéologie de la coopération dans son ensemble et le rôle joué par les ONG dans cette politique, comme en témoigne cette prise de position du parti nationaliste flamand: "S'il est incontestable que ces ONG fonctionnent grâce à beaucoup de bonne volonté, elles n'en sont pas moins exposées à l'interférence d'intérêts personnels et de motivations idéologiques et pratiquent parfois une certaine forme de "tourisme tiers-mondiste" (...). Reste à savoir en outre s'il est opportun de sous-traiter des projets à des ONG par le biais d'un financement quasi intégral. (...) On tente depuis longtemps de répandre l'idée selon laquelle les ONG travailleraient mieux et avec davantage d'idéalisme que les services officiels de coopération au développement. Rien n'est moins vrai. Les ONG n'échappent pas à l'amateurisme et à la naïveté, pas plus qu'à l'esprit de lucre, aux pratiques illégales et aux motifs idéologiques. Ainsi est-il frappant de constater qu'en Belgique,

13 Cf. De Morgen, 28 novembre 1995.

14 Chambre des représentants, Suivi des problèmes de l'Administration générale de la Coopération au développement. Rapport fait au nom de la commission spéciale, $\mathrm{n}^{\circ}$ 1123/1, 8 juillet 1997.

15 Il est à noter que MSF est une ONG humanitaire, et non une ONG de développement.

16 Ibidem, p. 19 et suivantes. 
on trouve une ou plusieurs ONG dans le giron de chaque famille politique, abstraction faite du Vlaams Blok» ${ }^{17}$.

Le rapport de la commission spéciale pointe cinq problèmes principaux qui, aux yeux des rapporteurs, ne pourront être dépassés que par une profonde refonte de la politique.

Premièrement, les objectifs de la coopération belge au développement manquent de précision. En l'occurrence, le rapport met en évidence un manque de vision à long terme et une volonté des ministres ou secrétaires d'État en charge de la coopération de "se mettre à l'honneur " ${ }^{18}$, négligeant ainsi le maintien d'une cohérence dans le temps entre politique et programmes. Quant aux choix géographiques d'intervention, le rapport met en exergue l'arbitraire et l'absence de justification. Il dénonce également une coopération paternaliste. Enfin, il estime que la coopération au développement a peu de succès auprès des citoyens belges. Selon les rapporteurs, ces éléments entraînent un manque de continuité dans la politique et de facto un morcellement de celle-ci.

Deuxièmement, la structure, l'organisation et le personnel de l'AGCD ne sont pas suffisamment adaptés à la multiplicité des tâches qui leur incombent. En réponse à la complexification qu'a connue l'AGCD en trente ans, celle-ci devrait prendre appui sur un cadre structurel renforcé et sur des fonctionnaires qualifiés et engagés de façon stable dans les programmes. Elle souffre d'un manque de hiérarchisation interne et semble être peu ouverte vers l'extérieur. Le rapport dénonce un manque de dialogue entre l'AGCD et les collaborateurs qui sont sur le terrain. Enfin, le document met en lumière le pouvoir du cabinet du ministre par rapport à celui de l'AGCD : « Les relations et la répartition des tâches entre l'AGCD et le cabinet constituent une part essentielle du problème. D'une part, l'administration se plaint que les cabinets se substituent bien trop souvent à l'administration. D'autre part, les cabinets se plaignent d'être contraints d'accomplir des tâches qui incombent en fait à l'administration " ${ }^{19}$.

Troisièmement, la prise de décision et les procédures appliquées au sein de la coopération belge au développement manquent de transparence et d'efficacité. La prise de décision est trop lourde, ce qui peut faire perdre beaucoup de temps à l'ensemble des acteurs. La pertinence des projets de développement est trop peu questionnée. Enfin, il n'existe pas de véritable méthode pour construire et suivre un projet de développement, alors que des outils sont disponibles par ailleurs depuis la fin des années 1960 (développés notamment par l'ONU et la BM).

Quatrièmement, le contrôle et l'évaluation de la coopération au développement ne sont pas efficaces et fonctionnels. Ce paramètre est intrinsèquement lié aux précédents, dans la mesure où le manque de suivi et d'évaluation est en partie dépendant de la surcharge de travail, causée elle-même par le manque d'effectif et par la lourdeur des procédures. De plus, la nécessité de développer une activité réflexive de suivi et d'évaluation a mis du temps avant de s'établir dans la coopération belge. Le rapport déplore le manque de contrôle sur la pertinence des dossiers et l'absence de culture d'évaluation au sein de l'AGCD : "La coopération belge au développement n'a pas l'habitude d'évaluer le contenu des projets et des programmes. C'est ainsi que, jusqu'il y a peu, seuls deux

\footnotetext{
Ibidem, p. 233-234.

Ibidem, p. 20.

Ibidem, p. 23.
} 
fonctionnaires étaient attachés au service Évaluation et Contrôle. (...) Une "mentalité de comptable" prédomine ${ }^{20}$. Or, selon la commission spéciale, l'évaluation est essentielle car la coopération est d'abord un projet politique et ne s'inscrit pas dans un marché classique dans lequel l'offre et la demande l'organisent, puisqu'il n'y a pas formellement de demande ${ }^{21}$.

Cinquièmement et enfin, les domaines respectifs de la coopération au développement et du commerce extérieur sont mal délimités. La commission spéciale dénonce plus particulièrement la pratique de prêts d'État à État dans le cadre de la coopération financière. La coopération et le commerce extérieurs ont des liens étroits; d'ailleurs, certains accords de coopération sont d'abord passés pour des raisons économiques. S'ajoute à cela la notion d' "aide liée ", qui implique que l'argent octroyé par l'État belge soit alloué à des biens ou à des services du pays donateur, en l'occurrence la Belgique. Par conséquent, le rapport prône une délimitation bien plus claire des compétences des relations économiques extérieures et de la coopération au développement à travers la notion le «déliement de l'aide » ${ }^{22}$ (c'est-à-dire le fait que l'argent versé dans le cadre de la coopération puisse être utilisé pour acheter des biens et des services auprès de n'importe quel pays).

Partant de ces constats qui font état d'une coopération belge affaiblie, la commission spéciale présente une série de recommandations.

Primo, le renforcement de la politique de coopération et de ses instruments est indispensable. Selon le rapport, il convient de mettre en place une vision politique claire, d'autant que le nombre d'engagements et de sommets internationaux ne cesse de croître. Il s'agit de renforcer la coopération belge pour répondre à ce champ qui se complexifie. Cela passe aussi par un renforcement des ressources humaines, tant sur le plan quantitatif que sur le plan qualitatif. Il s'agit aussi d'améliorer les liens entre les représentants de l'État belge dans les pays partenaires et l'AGCD.

Secundo, les objectifs de la coopération belge au développement doivent être spécifiés. Il s'agit d'apporter une vision stratégique à la coopération belge, définie en collaboration avec les pays partenaires de la Belgique.

Tertio, il faut opérer une concentration sectorielle et géographique. Il est ici question de poser une série de choix relatifs aux objectifs prédéfinis, en vue d'éviter la dispersion de l'aide, tant sur le plan des secteurs d'activité que sur celui des interventions géographiques. Selon la commission spéciale, la Belgique doit désormais se concentrer sur des pays dans lesquels le personnel est compétent et qualifié, là où il est possible de développer de l'expertise.

Quarto, le concept de cogestion doit être mis en pratique. Concrètement, il s'agit de collaborer davantage avec le pays partenaire et de cogérer avec lui les aspects liés aux programmes mis en œuvre ainsi que les modalités financières. Il convient qu'une loi

${ }^{20} \quad$ Ibidem, p. 27.

1 Il convient de souligner que, à cette époque, les activités d'évaluation de politiques publiques sont très peu développées en Belgique : il faudra attendre la fin des années 1990 pour voir émerger dans d'autres domaines politiques les exigences européennes en matière d'évaluation des programmes structurels (cf. S. JACOB, F. VARONE, Évaluer l'action publique. État des lieux et perspectives en Belgique, Bruxelles/ Gand, Politique scientifique fédérale, Academia Press, 2003).

Ce concept est soutenu par l'OCDE depuis la fin des années 1990. 
garantisse la continuité et pose les fondements juridiques de la coopération au développement.

Quinto, l'administration de la coopération au développement doit se limiter à la préparation, à l'évaluation et au contrôle de la politique. La commission spéciale propose de créer une instance autonome - en l'occurrence, la Coopération technique belge (CTB, cf. infra) - qui sera chargée de l'exécution de la coopération bilatérale directe (l'aide d'État à État). Cette recommandation soutient une prise de distance entre, d'une part, la gestion stratégique de la politique et, d'autre part, sa mise en œuvre.

\subsubsection{La réforme Moreels et ses effets}

Ces recommandations servent de base à la réflexion menant à la réforme (dite réforme Moreels, du nom du secrétaire d'État à la Coopération au développement R. Moreels), qui procède à une transformation majeure du secteur.

Adoptée quelques jours avant le terme du gouvernement Dehaene II, la loi du 25 mai 1999 relative à la coopération internationale belge ${ }^{23}$ dote la coopération belge d'un cadre légal. Cette loi permet de préciser les objectifs généraux de la coopération belge (tels que "le développement humain durable»), de définir les différents piliers de l'aide belge (coopération directe, indirecte et multilatérale) et d'apporter une vision politique à plus long terme.

La réforme génère un organigramme reposant sur trois pôles, en vue d'assurer une meilleure répartition des responsabilités entre : l'élaboration de la politique, la mise en œuvre de celle-ci sur le terrain (cf. infra) et l'évaluation. Premièrement, l'Administration générale de la Coopération au développement (AGCD, actuelle Direction générale du développement - DGD), intégrée au SPF Affaires étrangères, Commerce extérieur et Coopération au développement, est l'organe d'élaboration de la politique de la coopération belge au développement. Deuxièmement, la Coopération technique belge (CTB), instance autonome, a pour mandat d'exécuter la politique de coopération au développement pour le compte de l'État belge ${ }^{24}$. Il s'agit d'une société anonyme de droit public à finalité sociale, liée à l'État belge par un contrat de gestion. Jusqu'à cette scission, l'AGCD était en charge de toutes les étapes de la politique - de l'élaboration à la mise en ouvre - en matière de coopération au développement. Les deux nouvelles structures doivent collaborer pour la mise en œuvre de la coopération bilatérale directe (entre États partenaires). Troisièmement, le futur Service de l'évaluation spéciale (SES), qui répondra directement au Parlement, sera chargé de l'évaluation.

Dès le début, les relations entre l'AGCD/DGD et la CTB sont difficiles, et ce pour différentes raisons : de nombreux fonctionnaires de l'AGCD sont frustrés de n'avoir pas été retenus pour rejoindre la CTB et/ou ont le sentiment d'avoir été dépossédés de la matière noble de la coopération, à savoir les actions sur le terrain et la mise en œuvre; les trois premiers directeurs de la CTB adoptent des attitudes jugées

${ }_{24}^{23}$ Moniteur belge, $1^{\text {er }}$ juillet 1999.

24 Loi du 21 décembre 1998, portant création de la Coopération technique belge sous la forme d'une société de droit public, Moniteur belge, 30 décembre 1998. La CTB a le statut de société de droit public à finalité sociale (cf. infra). 
problématiques; les relations des directeurs généraux respectifs des deux structures sont faites d'animosité personnelle et idéologique. Les rapports entre les deux entités resteront longtemps conflictuels, notamment parce qu'elles s'inscrivent dans des logiques organisationnelles différentes. L'AGCD/DGD travaille selon un modèle bureaucratique d'administration, alors que la CTB fonctionne comme une entreprise privée avec davantage de flexibilité, même si elle est massivement contrôlée (commissaires du gouvernement, commissaire aux comptes, inspecteur des finances, audit interne).

Le mouvement de la réforme Moreels se poursuit en 2003, lorsque le gouvernement Verhofstadt I (VLD/PS/Fédération PRL FDF MCC/SP/Écolo/Agalev) - au sein duquel le secrétaire d'État à la Coopération au développement est Eddy Boutmans (Agalev) - crée le Service de l'évaluation spéciale (SES) ${ }^{25}$ puis désigne un évaluateur spécial pour le mettre en place et le diriger ${ }^{26}$. Le mandat du SES est d'évaluer les activités de coopération belge au développement et d'en rendre compte au Parlement fédéral en toute indépendance (cf. infra). En 2010, le SES absorbera en outre le service d'évaluation interne de la DGD ${ }^{27}$.

Par ailleurs, la réforme de mai 1999 entérine la légitimité des ONG belges (coopération bilatérale indirecte) en tant que structures représentatives de la société civile et engagées dans la lutte contre la pauvreté.

Le rapport de la commission parlementaire avait mis en exergue une série d'éléments relatifs au secteur des ONG. À cette époque, environ 80 ONG étaient agréées par l'État belge. Le secteur était caractérisé par une grande diversité, une importante disparité et peu de professionnalisme. L'AGCD devait gérer beaucoup de projets. Les procédures administratives étaient perçues comme lourdes. Il y avait un manque de contrôle et d'évaluation, tant sur le plan interne que sur le plan externe. Le rapport constatait également qu'il existait un problème d'entente entre l'AGCD et les ONG. Enfin, ce même document estimait qu'il serait préférable de davantage coordonner le travail des ONG et de concentrer l'aide.

Il importe de noter que les secrétaires d'État R. Moreels puis E. Boutmans soutiennent les ONG, de par leur sensibilité respective à la cause de celles-ci ${ }^{28}$. Les ONG se saisissent de cette situation pour revendiquer leur légitimité démocratique, compte tenu de leur rôle de soutien aux sociétés civiles de Belgique et des pays du Sud. Trois principes ont d'ailleurs émergé des débats de la commission spéciale : le droit d'initiative des ONG quant à la définition de leur propre programme à la demande d'un cofinancement ; le soutien du financement par programme (afin d'éviter l'approche par projet, qui accentue le morcellement du secteur); la réforme des instruments politiques (afin que la réglementation soit davantage en adéquation avec les réalités du terrain) ${ }^{29}$.

25 Arrêté royal du 17 février 2003 portant création d'un service Évaluation spéciale de la coopération internationale, Moniteur belge, 19 février 2003.

26 Arrêté royal du 4 avril 2003 portant à la désignation d'un évaluateur spécial auprès du service Évaluation spéciale de la coopération internationale, Moniteur belge, 2 mai 2003.

Arrêté royal du 25 février 2010 portant création d'un service Évaluation spéciale de la coopération internationale, Moniteur belge, 15 mars 2010.

28 Ainsi, R. Moreels a notamment fait partie des fondateurs de MSF.

29 Chambre des représentants, Suivi des problèmes de l'Administration générale de la Coopération au développement. Rapport fait au nom de la commission spéciale, $\mathrm{n}^{\circ}$ 1123/1, 8 juillet 1997. 
Ces principes ont donné lieu à des recommandations spécifiques au secteur des ONG. Primo, le financement par programme doit être généralisé et ce mouvement doit être accompagné par une évaluation en profondeur, notamment pour rétablir la relation de confiance entre les ONG et les pouvoirs publics. Cette généralisation du financement par programme ${ }^{30}$ implique le renforcement des ressources humaines à la DGD. Ces modifications doivent apparaître dans un nouvel arrêté royal et être mises en œuvre de façon graduelle. Secundo, il est nécessaire d'instaurer un dialogue politique permanent sur les grands axes politiques et sur le rôle des ONG dans l'aide publique au développement $(\mathrm{APD})^{31}$. Il s'agit par ailleurs de mener une réflexion sur la place des partenaires locaux et sur leurs relations avec les bureaux de représentants. Il importe aussi d'adopter une approche cohérente et de travailler en complémentarité avec les autres acteurs. L'assise sociale des ONG doit être renforcée. Tertio, il convient d'adopter une attitude d'ouverture compte tenu du renforcement du rôle des partenaires locaux et de favoriser la transparence dans la transmission d'informations du terrain vers la DGD, mais la DGD doit elle aussi faire preuve de plus de transparence et d'ouverture à l'égard du secteur des ONG. Quarto, les ONG doivent établir une comptabilité complète et transparente et ainsi améliorer leurs évaluations et leur contrôle. La future réglementation doit quant à elle permettre un report flexible des subsides non affectés. Les ONG devront assurer leur propre suivi-évaluation. Enfin, compte tenu des éléments présentés ci-dessus, les recommandations pointent la nécessité de renforcer le service ONG au sein de la DGD.

À la même époque, sur la scène internationale, les ONG deviennent également des actrices incontournables de la coopération au développement ${ }^{32}$. La participation des ONG à la gouvernance mondiale s'est intensifiée depuis le début des années 1970. Elles se développent en nombre, en taille et en diversité. Avec le temps, elles développent une capacité d'expertise qui leur permet de peser de façon plus marquée, notamment dans les domaines de l'environnement et des droits de l'homme. Cela est sans doute rendu possible aussi par une série de changements qui renforcent le secteur ONG international : la prise de conscience croissante et planétaire des problèmes mondiaux, l'arrêt de la Guerre froide, l'apparition de médias globaux permettant aux ONG d'être davantage connues du grand public, ou encore la diffusion des normes démocratiques.

À travers ce court historique, nous observons que, en une cinquantaine d'années, la coopération belge au développement a connu plusieurs réformes organisationnelles et politiques. À diverses reprises, les différents acteurs ont fait face à d'importants bouleversements politiques et institutionnels sur le plan national et international, faisant de la coopération belge le fruit d'oppositions et de consensus participant à l'équilibre parfois précaire d'un système en permanente mutation.

30 Une modalité «projet» a cependant été maintenue jusqu'en 2016 pour les plus petites organisations et pour des actions très ciblées et ponctuelles.

1 L'APD est constituée par « tous les apports de ressources qui sont fournis aux pays et territoires sur la liste des bénéficiaires d'APD, ou à des institutions multilatérales » (OCDE, «Is It ODA? », novembre 2008, www.oecd.org).

32 S. Charnovitz, «Les ONG : deux siècles et demi de mobilisation », L'Économie politique, n 13, 2002, p. 6-21 ; E. QUÉINNEC, «La croissance des ONG humanitaires. Une innovation devenue institution», Revue française de gestion, $\mathrm{n}^{\circ}$ 2007, 2007, p. 83-94. 


\section{LES ÉVOLUTIONS RÉCENTES DE LA COOPÉRATION AU DÉVELOPPEMENT : VERS UNE MANAGÉRIALISATION ET UNE COMPLEXIFICATION}

Ce deuxième chapitre fait brièvement état de l'évolution de la coopération au développement depuis les années 1980, et particulièrement de la façon dont les instances internationales ont influencé les débats. En effet, les interventions de trois acteurs majeurs en matière de coopération au développement se croisent et s'interpénètrent : celles des agences de Bretton Woods (c'est-à-dire la BM et le FMI), celles de l'ONU et celles de l'OCDE.

\subsection{LES CONCEPTIONS DE LA BANQUE MONDIALE ET DU FONDS MONÉTAIRE INTERNATIONAL}

Entre 1980 et 1990, le volontarisme est au cœur de la démarche de coopération. L'intervention du secteur public se veut renforcée, mais les agences internationales - à commencer par la BM et le FMI, qui dominent alors les débats en la matière préconisent l'ingérence du secteur privé dans l'économie des pays en développement et fixent des conditionnalités essentiellement économiques aux pays en développement (cf. les plans d'ajustement structurel - PAS).

Entre la fin des années 1980 et le début 2000, les instances internationales complètent leurs exigences en mobilisant les principes de «bonne gouvernance» et de démocratie. Désormais, les bailleurs ne ferment plus les yeux sur les conditions de gouvernance douteuses de certains pays, car les critères de démocratie deviennent une condition d'aide à part entière. Les conditionnalités économiques sont complétées par des conditionnalités politiques orientées sur la «bonne gouvernance » faisant place à une série d'engagements internationaux. 


\subsection{LES CONCEPTIONS DE L'ORGANISATION DES NATIONS UNIES}

Lors de la décennie 1990, face aux nombreuses contestations notamment de la société civile, l'ONU entreprend une réflexion sur l'efficacité de l'aide. Les Nations unies reviennent ainsi sur le devant de la scène internationale en matière de développement, alors que celle-ci était dominée depuis vingt ans par la BM et le FMI ${ }^{33}$.

Signée à New York le 8 septembre 2000, la Déclaration du millénaire des Nations unies est le fruit d'une décennie de réflexion réinitiée par l'ONU à travers une série de sommets mondiaux ${ }^{34}$. Elle est assortie d'un cadre opérationnel : les Objectifs du millénaire pour le développement (OMD). La stratégie onusienne vise huit objectifs quantitatifs ${ }^{35}$, dix-huit cibles claires et une quarantaine d'indicateurs chiffrés pour lutter contre l'extrême pauvreté ; l'horizon fixé est celui de l'année 2015. Toutefois, en 2015, les États engagés dans le processus des OMD sont forcés de constater que les huit objectifs n'ont pas été totalement atteints, même si cet engagement mondial a par exemple réduit en partie l'extrême pauvreté dans le monde (elle est passée de $47 \%$ en 1990 à $14 \%$ en $2015^{36}$ ). Ces résultats sont toutefois à relativiser, notamment au regard du développement socio-économique de l'Asie ${ }^{37}$. La principale plus-value des OMD a été l'engagement généralisé de la part des États signataires. En effet, ils « ont offert aux sociétés civiles de tous les pays un instrument de mesure et de contrôle des politiques publiques, et ont été à l'origine de grandes campagnes de mobilisation ${ }^{38}$.

Dès 2012, un nouveau processus participatif entre les États signataires permet l'élaboration d'un nouvel engagement international universel (couvrant les années 2015-2030) : les Objectifs pour le développement durable (ODD), qui ont pour ambition d'aller plus loin que les OMD et de corriger leurs faiblesses. Les ODD se composent des « $5 \mathrm{P}$ » considérés comme des thématiques transversales : peuples, planète, prospérité, paix et partenariat. Les ODD intègrent les questions économiques et environnementales dans le prolongement des réflexions de la Conférence des Nations unies sur le développement durable dite Rio+20 (Rio de Janeiro, 20-22 juin 2012) et de la Conférence des parties à la Convention-cadre des Nations unies sur les changements climatiques devant se tenir à Paris en 2015 (la future COP21, alors en préparation). Ainsi, le développement durable devient un des nouveaux paradigmes de l'aide au développement promu par les Nations unies et influence le contenu des politiques nationales et internationales en la matière.

33 F. LAPEYRE, «Objectifs du millénaire pour le développement : outils de développement ou cheval de Troie des politiques néolibérales?", Alternatives Sud, volume 13, $n^{\circ} 1$ : B. DUTERME (dir.), Objectifs du millénaire pour le développement, 2006, p. 9-26.

34 Ibidem.

35 Réduire l'extrême pauvreté et la faim ; assurer l'éducation primaire pour tous ; promouvoir l'égalité des sexes et l'autonomisation des femmes; réduire la mortalité des enfants de moins de 5 ans ; améliorer la santé maternelle ; combattre le $\mathrm{VIH} / \mathrm{sida}$, le paludisme et d'autres maladies; assurer un environnement durable; mettre en place un partenariat mondial pour le développement.

D. VERGER, «2015: une année historique pour le développement », Études. Revue de culture contemporaine, $\mathrm{n}^{\circ} 12,2015$, p. 19-29.

37 J.-R. Chaponnière, M. LAUTIER, «La montée des échanges Sud-Sud dans le commerce mondial », in L'économie mondiale 2013, Paris, La Découverte, 2012, p. 91-103.

38 D. Verger, « 2015 : une année historique pour le développement », op. cit. 


\subsection{LES CONCEPTIONS DE L'ORGANISATION DE COOPÉRATION ET DE DÉVELOPPEMENT ÉCONOMIQUES}

Le 2 mars 2005, lors d'un forum de haut niveau, les ministres des pays développés et en développement signent la Déclaration de Paris sur l'efficacité de l'aide au développement. Il s'agit d'une réforme des modalités de gestion de l'aide pilotée cette fois-ci par le Comité d'aide au développement (CAD, organe de l'OCDE). Le texte de la Déclaration indique : «Si la réalisation de ces objectifs suppose un accroissement des volumes d'aide et d'autres ressources affectées au développement, une augmentation significative de l'efficacité de l'aide est également nécessaire pour soutenir les efforts déployés par les pays partenaires en vue de renforcer la gouvernance et d'améliorer les résultats obtenus sur le front du développement». Cette Déclaration marque un changement dans la manière de concevoir l'aide. Il n'est désormais plus question d'une coopération articulée autour de bonnes volontés, mais d'une coopération orientée sur la performance, prônant ainsi une gestion axée sur les résultats pour augmenter les effets dits positifs de l'aide.

Différents principes sont au cœur de la Déclaration de Paris. Primo, l'appropriation : les pays du Sud doivent exercer une maitrise de leur politique et de leur stratégie de développement. Secundo, l'harmonisation : les actions des pays donateurs doivent être harmonisées et davantage transparentes, permettant ainsi une efficacité collective. Tertio, l'alignement: les pays donateurs doivent faire reposer leur soutien sur les stratégies nationales de développement des pays partenaires. Quarto, la responsabilité mutuelle : les pays donateurs et partenaires doivent être mutuellement responsables de l'efficacité de l'aide. Quinto, la gestion axée sur les résultats (GAR) : il s'agit d'un modèle managérial visant une gestion efficace des ressources, en vue d'améliorer les processus et d'atteindre de meilleurs résultats dans le secteur public.

Une lecture critique de cette Déclaration de Paris met en évidence que deux de ces principes présentent un fort pouvoir normatif : d'une part, la GAR soutient des concepts au caractère managérial, puisqu'il s'agit désormais de produire des résultats qui répondent aux objectifs préalablement fixés ; d'autre part, l'harmonisation implique que l'ensemble des acteurs du Nord et du Sud se soumettent aux mêmes principes de la Déclaration, ce qui constitue une forme d'universalisme dans lequel les spécificités socio-contextuelles sont gommées au profit d'un seul et même mode de pensée, orienté vers la performance, celle-ci semblant être devenue la norme ${ }^{39}$.

La Déclaration de Paris s'inscrit dans une série de forums de haut niveau organisés entre 2003 et 2016 et réunissant les États membres de l'OCDE. Ces différents forums de haut niveau peuvent être classés en trois catégories : primo, le financement du développement (Monterrey en 2002, Doha en 2008, Addis Abeba en 2015) ; secundo, l'efficacité de l'aide (Rome en 2003, Paris en 2005, Accra en 2008, Busan en 2011), qui oriente la coopération internationale sur la notion d'États fragiles; tertio, le partenariat global pour une coopération plus efficiente (Mexico en 2014, Nairobi en 2016). Chacune de ces réunions

39 C. SAliba-Couture, «L'appropriation et la Déclaration de Paris. Un discours convaincant, mais en sommes-nous convaincus? ", Revue canadienne d'études du développement, volume $32, \mathrm{n}^{\circ} 2,2011$, p. 180-195. 
confirme l'intérêt d'une coopération plus efficace et efficiente et l'importance de maintenir l'aide publique au développement.

\subsection{SYNTHÈSE : TROIS POINTS DE VUE SUR LA COOPÉRATION AU DÉVELOPPEMENT}

Au fil du temps, trois acteurs se partagent donc le pouvoir en matière d'idéologie du développement. Jusque dans les années 1990, la BM et le FMI sont dominants et insufflent une vision résolument économique du développement, dans la veine du manifeste de W. W. Rostow. À partir de la fin des années 1990, l'ONU reprend la main par le biais des OMD puis des ODD : le paradigme dominant devient la lutte contre la pauvreté à travers une série de priorités telles que la santé et l'éducation, et ensuite le développement durable. Avec l'émergence du nouveau millénaire, c'est l'OCDE qui devient l'acteur dominant pour la coopération au développement en soutenant des politiques de qualité, de performance, d'efficacité, d'harmonisation et de bonne gestion : des principes qui président aujourd'hui à l'organisation de la coopération au développement. La Déclaration de Paris marque un tournant majeur dans la manière de penser la coopération internationale. Elle est mobilisée par les acteurs internationaux et par les autorités nationales comme un engagement international percolant jusqu'au niveau des pratiques et de la base de la chaîne du développement jusqu'aux ONG. Ainsi, la performance et l'efficacité sont devenues les maîtres mots au sein de la coopération au développement. En Belgique, ce nouveau paradigme est pris en considération dans l'élaboration des réformes et des textes de loi (cf. infra). 


\section{LES ACTEURS DE LA COOPÉRATION BELGE : ÉTAT DES LIEUX ET ÉVOLUTIONS}

Le deuxième chapitre a montré l'influence du cadre international depuis les années 1990, indiquant le poids qu'ont eu les différents acteurs internationaux sur la coopération d'un point de vue idéologique et l'influence que cela a eue dans la pratique.

Pour sa part, le présent chapitre dresse un état des lieux des différents acteurs de la coopération belge au développement, en les présentant sur les plans structurel, organisationnel et budgétaire. La coopération belge étant organisée en trois piliers (multilatéral, bilatéral et non gouvernemental), nous avons opté pour cette structure. Nous nous arrêtons donc tout d'abord sur la coopération multilatérale, c'est-à-dire celle mise en œuvre par les agences internationales. Ensuite, nous nous penchons sur les acteurs de la coopération bilatérale directe (gouvernementale) : la Direction générale du Développement (DGD), l'Agence belge de développement (Enabel, anciennement dénommée Coopération technique belge - CTB), le Service de l'évaluation spéciale (SES) et BIO-Invest. Enfin, nous présentons les acteurs non gouvernementaux : d'une part, les ONG de développement, qui sont étudiées de manière spécialement approfondie, et, d'autre part, les acteurs hors ONG (établissements d'enseignement supérieur, institutions scientifiques, instituts de formation, villes et communes, syndicats). Par ailleurs, nous évoquons également les acteurs dits périphériques.

Dans la lignée du précédent chapitre, cette troisième partie souligne combien et comment, avec le temps, tous les acteurs de la coopération belge ont évolué, jusqu'à aborder la réforme actuellement à l'œuvre.

\subsection{PRÉAlABLE : LES TROIS PILIERS DE LA COOPÉRATION BELGE}

La coopération belge au développement est structurée en trois piliers : la coopération multilatérale, la coopération bilatérale (gouvernementale) et la coopération non gouvernementale, auxquelles s'ajoutent les acteurs périphériques.

La coopération multilatérale est mise en œuvre par les agences internationales telles que le Programme des Nations unies pour le développement (PNUD), la Banque mondiale (BM) ou l'Union européenne. L'État belge soutient financièrement ces agences, qui interviennent pour le compte des États membres, dont la Belgique. 
La coopération bilatérale (gouvernementale) a lieu entre l'État belge et les États avec lesquels il signe des accords de coopération. La DGD est l'administration en charge de l'aspect stratégique de la politique de coopération. Enabel est son partenaire immédiat : principal intermédiaire, elle sert de structure de mise en œuvre de la coopération directe. Par ailleurs, deux structures sont assimilées à la coopération gouvernementale : le SES, qui est l'organe chargé de l'évaluation, et BIO-Invest, qui soutient les investissements du secteur privé dans les pays partenaires au Sud.

La coopération non gouvernementale est mise en œuvre par les acteurs de la coopération non gouvernementale (ACNG): ONG, établissements d'enseignement supérieur, institutions scientifiques, instituts de formation, villes et communes, syndicats. Ces acteurs indirects sont principalement financés par les structures étatiques et interviennent eux aussi dans les pays partenaires de la Belgique. Ils jouent un rôle opérationnel important et ont pour la plupart des connexions directes avec la société civile locale au Nord et au Sud.

À côté de ces trois piliers, se placent les acteurs périphériques: B-FAST, qui est l'organe de mise en œuvre de l'aide d'urgence, et le Fonds belge pour la sécurité alimentaire (FBSA), qui œuvrait jusqu'il y a peu pour les questions d'accès à l'alimentation dans le monde.

\subsection{LA COOPÉRATION MULTILATÉRALE}

La coopération multilatérale est constituée des contributions à charge du budget de la Coopération belge au développement, versées aux organisations multilatérales et destinées aux interventions de celles-ci dans le domaine de la coopération au développement. Elle est définie de la manière suivante : "programmes ou projets financés par l'État belge et exécutés par une organisation internationale et des contributions belges à des organisations internationales pour les programmes ou projets de développement». Les objectifs de la coopération multilatérale sont en adéquation avec ceux de la coopération belge. Le budget pour l'année 2016 était de 329 millions d'euros, soit un peu plus de $20 \%$ du budget annuel de l'aide publique au développement (APD) belge.

La liste des organisations partenaires de l'État belge est la suivante ${ }^{40}$ :

- l'Entité des Nations unies pour l'égalité des sexes et l'autonomisation des femmes (ONU Femmes);

- le Fonds mondial de Lutte contre le sida, la tuberculose et le paludisme (GFATM);

- le Fonds des Nations unies pour l'enfance (UNICEF) ;

- le Fonds des Nations unies pour la population (FNUAP);

- le Groupe de la Banque mondiale, en ce compris :

- l'Association internationale de développement (IDA),

- la Banque internationale pour la reconstruction et le développement (BIRD),

- le Fonds africain de développement (FAD),

40 Arrêté royal du 29 mai 2015 déterminant les partenaires de la coopération multilatérale, Moniteur belge, 15 juin 2015. 
- le Fonds pour l'environnement mondial (FEM),

- l'Initiative d'allègement de la dette multilatérale (IADM),

- l'Initiative Pays pauvres très endettés (PPTE),

- le Partenariat mondial pour l'éducation (PME);

- le Groupe consultatif de recherche agronomique internationale (GCRAI) ;

- le Haut-Commissariat des Nations unies aux droits de l'Homme (HCDH);

- le fonds fiduciaire pour le développement des capacités « Mobilisation des recettes » du FMI (MR, anciennement «Politique et administration fiscales »);

- l'Organisation internationale pour les migrations (OIM);

- l'Organisation internationale du travail (OIT);

- l'Organisation mondiale de la santé (OMS);

- l'Organisation des Nations unies pour l'alimentation et l'agriculture (FAO);

- le Programme commun des Nations unies sur le VIH/sida (ONUSIDA);

- le Programme des Nations unies pour le développement (PNUD);

- le Programme des Nations unies pour l'environnement (PNUE).

Récemment, un arrêté royal a disposé que les ministres fédéraux des Finances et de la Coopération au développement exercent désormais conjointement la tutelle sur la représentation belge dans les différents organes du Groupe de la Banque mondiale (compétence qui appartenait jusqu'alors exclusivement au ministre des Finances) ${ }^{41}$. Cette décision a pour objectif, d'une part, de combiner l'expertise du pays en matière de coopération au développement et celle en matière financière et bancaire et, d'autre part, de permettre à la Belgique d'exercer davantage d'influence sur la politique de la BM. En effet, la BM est aujourd'hui le principal partenaire multilatéral de la politique belge de développement et le principal acteur mondial dans le domaine du développement international.

\subsection{LA COOPÉRATION BILATÉRALE DIRECTE (GOUVERNEMENTALE)}

D'après la définition qui en est donnée dans la loi du 25 mai 1999, la coopération bilatérale directe (gouvernementale) consiste dans les "programmes ou projets dans un pays partenaire, financés par l'État belge, sur base d'une convention entre les deux pays ». Elle concerne les relations de coopération entre la Belgique et ses États partenaires, avec lesquels elle signe des programmes de coopération. Récemment, l'État belge a recentré son aide autour de quatorze pays considérés comme des États fragiles ${ }^{42}$ : Bénin, Burkina

41 Arrêté royal du 24 novembre 2016 relatif à la tutelle sur plusieurs institutions financières internationales, Moniteur belge, 5 décembre 2016. Ce texte abroge et remplace l'arrêté royal du 12 novembre 2001 relatif à la tutelle de la Banque internationale pour la reconstruction et le développement, de l'Association internationale de développement, de la Banque asiatique de développement, du Fonds asiatique de développement, de la Banque africaine de développement et du Fonds africain de développement (Moniteur belge, 20 décembre 2001).

42 La définition des États fragiles par l'OCDE demeure minimale : « Un État est fragile lorsque les instances étatiques n'ont pas la volonté et/ou la capacité d'assumer des fonctions essentielles requises pour faire 
Faso, Burundi, République démocratique du Congo, Guinée, Mali, Maroc, Mozambique, Niger, Ouganda, Palestine, Rwanda, Sénégal et Tanzanie ${ }^{43}$.

La coopération directe représente $29 \%$ du budget annuel de la coopération belge ${ }^{44}$, soit approximativement 332 millions d'euros pour l'année 2016, dont plus de la moitié va directement à Enabel.

\subsubsection{La Direction générale du Développement (DGD)}

La DGD du SPF Affaires étrangères, Commerce extérieur et Coopération au développement est chargée d'élaborer la politique et la stratégie belge de coopération avec le ministre fédéral en charge de la Coopération au développement. Compte tenu de la réforme actuelle, elle voit son rôle davantage se recentrer sur les aspects stratégiques de la coopération. Elle vient de connaître une réorganisation interne en cinq grandes directions.

La direction géographique (D1) se concentre sur le contexte géographique, à partir d'un angle d'approche régional qui tient compte de la spécificité du contexte et des besoins des pays du Sud, et dont l'objectif est l'obtention des résultats de développement. La D1 est composée d'une cellule et de deux services: D1.1 (Cellule administrative et financière), D1.2 (Afrique de l'Ouest et Monde arabe) et D1.3 (Afrique centrale et australe).

La direction thématique (D2) est responsable de la définition stratégique, de l'utilisation et du suivi des instruments belges de développement thématique pour l'obtention de résultats de développement. La D2 se compose d'une cellule et de quatre services: D2.0 (Cellule administrative), D2.2 (Croissance inclusive), D2.3 (Développement social), D2.4 (Climat, environnement, ressources naturelles) et D2.5 (Consolidation de la société) ${ }^{45}$. S'y ajoute en outre un service D2.PCD (Cohérence des politiques pour le développement).

La direction Société civile (D3) s'occupe des relations avec les acteurs non gouvernementaux de la politique belge de coopération: les ONG, les établissements d'enseignement supérieur, les institutions scientifiques, les instituts de formation, les villes et communes, et les syndicats. Ces organisations jouent un rôle important en matière de lutte contre la pauvreté, de développement des capacités et d'appui critique à la politique dans la mesure où elles entretiennent des relations de proximité avec les bénéficiaires. Elle est constituée d'une cellule et de quatre services : D3.0 (Suivi des subsides aux acteurs non gouvernementaux), D3.1 (Éducation au développement), D3.2 (Afrique du Nord et de l'Ouest, Monde arabe), D3.3 (Afrique centrale et australe) et D3.4 (Asie et Amérique latine).

reculer la pauvreté et promouvoir le développement, et d'assurer la sécurité et le respect des droits de l'homme de la population " (cité par F. GAULmE, " "États faillis", "États fragiles" : concepts jumelés d'une nouvelle réflexion mondiale ", Politique étrangère, $n^{\circ} 1,2011$, p. 17-29).

Arrêté royal du 29 mai 2015 déterminant les pays partenaires de la coopération gouvernementale, Moniteur belge, 15 juin 2015.

44 Chiffre de l'APD belge, 2016.

45 Existait précédemment la cellule D2.1 (Aide humanitaire) ; elle a été supprimée suite à la création de la D5. 
La direction Gestion de l'organisation (D4) soutient la mission stratégique de la DGD et se concentre sur la qualité et sur l'efficacité de l'aide, ainsi que sur la communication et la redevabilité. Un intérêt particulier est apporté à la réalisation de résultats de développement, à la reddition de comptes, au partage des enseignements tirés, ainsi qu'au développement d'un contrôle interne adéquat et de gestion des risques. Elle est composée d'une cellule et de deux services : D4.0 (Cellule administrative et financière), D4.1 (Gestion générale) et D4.2 (Gestion de la qualité et résultats).

La direction Aide humanitaire et transition (D5) est compétente pour l'action humanitaire et le développement transitionnel. Sa création est motivée, d'une part, par l'ampleur que représente l'action humanitaire en termes d'activités et de budget et, d'autre part, par la nécessité de regrouper de manière plus cohérente le savoir-faire et les instruments qui s'adressent aux situations dites « de transition». La D5 se constitue de deux services: D5.1 (Aide humanitaire) et D5.2 (Développement transitionnel et gouvernance).

Outre ces cinq directions, la DGD compte un comité stratégique, qui a pour mission de promouvoir la cohérence interne de la politique belge de développement, de développer les propositions en matière de politique adressées au ministre. Ce comité est aussi chargé du dialogue institutionnel avec les différents partenaires de la coopération belge au développement. La DGD compte enfin le Service de l'envoyé spécial Asile et Migration (ESMA).

\subsubsection{L’Agence belge de développement (Enabel)}

Enabel - nouvelle appellation de la Coopération technique belge (CTB) depuis 2018 exécute la politique de coopération au développement pour le compte de l'État belge. Il s'agit d'une société anonyme de droit public à finalité sociale, liée à l'État belge par un contrat de gestion.

Le siège social d'Enabel se trouve à Bruxelles, mais la structure possède un bureau de représentation dans chacun des pays partenaires de la Belgique. En matière de personnel, le siège compte 181 personnes ${ }^{46}$. Les ressources humaines du siège sont principalement composées de gestionnaires et d'experts (selon les pays et/ou thématiques). Structurée en quatre directions (ressources humaines; opérations; thématiques ; finances), Enabel compte 246 personnes expatriées et 1073 employés locaux. Les États dits fragiles représentent $61 \%$ de ses missions et c'est en Afrique qu'Enabel est la plus active. Son budget annuel est de 217 millions d'euros ${ }^{47}$, dont $90 \%$ sont alloués à la coopération gouvernementale belge.

Enabel inscrit son action selon le cadre légal, selon les lignes directrices définies par le ministre et son administration, et selon le contrat de gestion qui la lie à l'État belge. Enabel est active dans cinq secteurs clés : les soins de santé, l'agriculture, les infrastructures de base, l'enseignement et la gouvernance. Ces secteurs sont issus des neuf priorités thématiques et sectorielles de la coopération belge au développement : les droits humains,

${ }_{47}$ Les chiffres de cette partie sont issus de CTB, « Rapport annuel », 2016.

Plus de 180 millions d'euros proviennent de l'APD belge. Les $30 \%$ restants du budget de la coopération gouvernementale sont distribués parmi d'autres acteurs et infrastructures liés à ce pilier. 
le travail décent, la consolidation de la société, la dimension genre, la protection de l'environnement, les soins de santé, l'enseignement et la formation, l'agriculture, et les infrastructures de base. En adhérant à l'International Aid Transparency Initiative (IATI) en 2012, Enabel (alors CTB) a pris l'engagement d'accroître la transparence de son aide et a ouvert une plateforme d'information: Open.Enabel (anciennement, Open.CTB).

À côté de ces principales lignes d'intervention dans les pays cibles, Enabel est également active en Belgique à travers différents programmes. En matière d'éducation au développement, elle est responsable du projet "Annoncer la couleur / Kleur bekennen », qui est en charge de l'éducation à la citoyenneté mondiale. L'Info-cycle était, jusqu'à sa disparition en septembre 2017, un autre programme de sensibilisation et de formation à la coopération au développement et aux enjeux mondiaux. Le Programme Junior organise l'envoi de jeunes coopérants dans des projets au sein des pays partenaires, soit par le biais d'Enabel soit via une ONG, afin qu'ils disposent d'une expérience professionnelle dans le Sud. Enfin, Enabel coordonne le Trade for Development Center (TDC, anciennement dénommé Fair Trade Center), qui promeut les produits équitables et durables en Belgique, notamment à travers la coordination de la campagne annuelle " semaine du commerce équitable».

La CTB a été créée en 1998 avec le statut de société anonyme de droit public à finalité sociale, dont le seul actionnaire est l'État belge ${ }^{48}$. Suite à une décision du Conseil des ministres du 15 avril 2016, la CTB est devenue l'Agence belge de développement (Enabel) à partir du $1^{\text {er }}$ janvier $2018^{49}$. Ce glissement vers le statut d'agence implique une série de changements confirmant un processus de mise en œuvre du "new public management » au niveau belge. Comme l'explique Béatrice Van Haeperen (UCL et IWEPS), «l'une des manifestations les plus visibles de la transformation structurelle des services publics est leur déconcentration: si les responsabilités stratégiques restent entre les mains de l'administration centrale, l'exécution des politiques est de plus en plus souvent confiée à des entités autonomes, généralement désignées par le terme d’agences (...). Les agences sont dotées d'une personnalité juridique propre, disposent d'un domaine de compétences particulier et jouissent d'une certaine indépendance opérationnelle ${ }^{50}$.

Enabel est liée à l'État belge par un contrat de gestion. Dans les faits, le quatrième contrat de gestion, conclu en avril $2014^{51}$, n'a pas été véritablement appliqué. Les négociations liées à ce quatrième contrat ont en effet été longues et pénibles pour les parties prenantes.

48 Loi du 21 décembre 1998, portant création de la Coopération technique belge sous la forme d'une société de droit public, Moniteur belge, 30 décembre 1998.

49 Loi du 23 novembre 2017 portant modification du nom de la Coopération technique belge et définition des missions et du fonctionnement de Enabel, Agence belge de développement, Moniteur belge, 11 décembre 2017.

50 B. VAN HAEPEREN, «Que sont les principes du new public management devenus? Le cas de l'administration régionale wallonne", Reflets et perspectives de la vie économique, volume 51, $\mathrm{n}^{\circ}$ 2, 2012, p. 84.

51 Quatrième contrat de gestion du 9 avril 2014 entre l'État belge et la société anonyme de droit public à finalité sociale Coopération technique belge (CTB), Moniteur belge, 10 juin 2014. Le troisième contrat de gestion datait du 2 août 2006 (Moniteur belge, 27 septembre 2006); des arrêtés royaux l'avaient modifié les 21 août 2008 (Moniteur belge, 17 septembre 2008) et 20 juillet 2012 (Moniteur belge, 30 novembre 2012), puis prolongé le 8 janvier 2013 (Moniteur belge, 20 février 2013). Quant à eux, les deux premiers contrats de gestion dataient respectivement du 23 avril 1999 (Moniteur belge, 28 mai 1999) et du 15 octobre 2002 (Moniteur belge, 21 novembre 2002). 
La durée du processus ${ }^{52}$ a joué en défaveur de sa mise en œuvre, à tel point que la réforme actuelle a rendu nécessaires de nouvelles négociations. Le cinquième contrat de gestion a été arrêté le 17 décembre 2017 ; entré en vigueur le $1^{\text {er }}$ janvier 2018, il doit couvrir les années 2018-2022 ${ }^{53}$.

Dorénavant, Enabel dispose de davantage d'autonomie opérationnelle, tant sur le terrain qu'en Belgique. Le représentant d'Enabel est directement attaché à l'ambassade belge dans le pays partenaire. L'agence a également davantage de connexions avec les partenaires au Sud, puisque sa position de bailleur est renforcée par son autonomie financière accrue. Enabel consolide aussi ses rapports avec d'autres bailleurs de fonds que l'État belge, comme l'Union européenne, confortant encore son autonomie financière. Elle est d'ailleurs reconnue comme " seventh pillar assessment " par cette dernière ${ }^{54}$.

En Belgique, le ministre peut désormais adresser une demande directe d'intervention à Enabel, via une lettre de mission, réduisant la charge administrative pour la DGD, et donnant ainsi davantage d'autonomie opérationnelle à Enabel.

\subsubsection{Le Service de l'évaluation spéciale (SES)}

La création du SES est l'un des effets de la crise de la fin des années 1990 (cf. supra). Institué en 2003, le SES est habilité à évaluer toutes les activités de l'État belge reconnues comme aide publique au développement par l'OCDE. Sa principale mission est de planifier, d'exécuter et d'assurer le suivi des évaluations. Il doit rendre compte de son action auprès du Parlement fédéral quant à la politique menée et à l'affectation des moyens dans la coopération au développement. Il rédige des conclusions et formule des recommandations en vue d'améliorer et d'adapter la politique. Le SES participe, rarement, à des évaluations internationales communes. En plus de cette fonction de reddition des comptes pour la coopération, il renforce a minima les capacités en évaluation des pays partenaires de la coopération belge, car il est très peu présent sur le terrain.

Le SES est extérieur à l'organigramme du SPF Affaires étrangères, Commerce extérieur et Coopération au développement. Cette position lui apporte une forme d'indépendance vis-à-vis de la hiérarchie administrative et politique mais, inévitablement, elle entraîne potentiellement un manque de connexion et de visibilité vis-à-vis des autres acteurs de la coopération. Si le SES possède une certaine indépendance institutionnelle, son indépendance opérationnelle est moindre. D'une part, parce qu'il dispose d'un budget limité qui est géré par la DGD et donc le ministre. D'autre part, l'évaluateur spécial, qui dirige le SES, n'a pas la main sur le recrutement de son personnel (puisque celui-ci est opéré via le Selor, organe du SPF Stratégie et Appui). Par conséquent, il ne peut pas engager les profils qu'il souhaite, en vue de maximiser les compétences de son service.

52 Le processus a notamment été ralenti par le fait que le gouvernement fédéral a été en affaires courantes durant 540 jours en 2010-2011 et par des relations interpersonnelles conflictuelles.

3 Premier contrat de gestion du 14 décembre 2017 entre l'État fédéral et la société anonyme de droit public à finalité sociale Enabel, Agence belge de développement, Moniteur belge, 22 décembre 2017.

Le pillar assessment est un système d'audit de la Commission européenne, basé sur sept piliers en lien avec le système de contrôle interne. 
Les membres du personnel du SES disposent d'une expérience variable dans la coopération au développement, mais aucun d'entre eux n'est évaluateur. L'équipe du SES est chargée de conduire et de gérer les évaluations et non de les mettre en œuvre. Pour ce faire, elle fait appel à des bureaux de consultance externes via des marchés publics. De même, contrairement à d'autres organes d'évaluation, le SES ne possède pas ses propres normes d'évaluation. Il travaille avec les «bonnes pratiques» établies par le CAD ${ }^{55}$. Celles-ci sont organisées autour de quatre grands principes : l'impartialité, l'indépendance, la crédibilité et la pertinence.

Par ailleurs, le SES s'inscrit dans le paradigme interprétatif de l'évaluation ${ }^{56}$, c'est-à-dire qu'il défend une vision pédagogique de son travail. Il envisage l'évaluation comme un accompagnement dont l'objectif ultime est d'éduquer et d'éclairer le commanditaire. Cette posture suscite des réticences de la part de la DGD, qui se positionne à mi-chemin entre le paradigme interprétatif et positiviste, en se montrant davantage soucieuse de mesurer les effets de l'intervention de manière objective et en mobilisant une hypothèse causale du changement social pour légitimer son action et ses choix politiques. Les différentes parties prenantes externes ont d'ailleurs des points de vue relativement différents sur les finalités de l'évaluation ${ }^{57}$. Le cabinet du ministre et l'administration s'inscrivent dans une conception orientée de reddition des comptes, alors qu'Enabel conçoit l'évaluation davantage comme une source d'apprentissage et d'amélioration de ses pratiques ${ }^{58}$. Dans les faits, le SES se trouve dans une position intermédiaire, compte tenu de l'obligation légale de satisfaire la demande du ministre en matière de reddition de comptes, tout en soutenant une vision axée sur l'apprentissage que réclament les acteurs de terrain en charge des programmes.

Le SES dispose d'un budget d'environ 1200000 euros par an, soit environ 0,1 \% du budget annuel de la coopération belge. Certaines parties prenantes considèrent néanmoins le budget comme étant trop important au regard de l'utilité des travaux du SES. A contrario, d'autres considèrent ses moyens comme trop faibles pour remplir les missions qui lui sont assignées ${ }^{59}$. Sur le plan des capacités humaines, le SES comptait en 2013 sept personnes pour assurer le suivi de deux à trois processus évaluatifs annuels. Aujourd'hui, cinq personnes composent le SES et un nouvel évaluateur spécial a été nommé en 2016 pour un mandat de six ans ${ }^{60}$.

En vertu de l'arrêté royal du 25 avril 2014, le SES est chargé d'apporter un appui technique à la DGD pour l'examen préalable des systèmes de suivi et d'évaluation

55 Comité d'aide au développement, « Normes de qualité pour l'évaluation du développement », avril 2010, www.oecd.org.

D. Aubin, C. DE VISSCHER, A. TROSCH, « Des objectifs communs, mais une démarche spécifique : l'évaluation par rapport aux autres outils de contrôle», in L. Albarello, D. Aubin, C. Fallon, B. VAN Haeperen (dir.), Penser l'évaluation des politiques publiques, Bruxelles, De Boeck, 2016, p. 17-37.

57 J. CONTOR, «L'évaluation des politiques publiques dans le champ de la coopération au développement belge : le cas du Service de l'évaluation spéciale ", Pyramides. Revue du Laboratoire d'études et de recherches en administration publique, $\mathrm{n}^{\circ} 28$ : A. PIRAux, D. PIRON (dir.), Les ressources publiques, 2017, en ligne.

58 Ibidem.

59 Ibidem.

60 Arrêté royal du 8 juin 2016 portant désignation de l'évaluateur spécial, Moniteur belge, 22 juin 2016. Cette nomination s'est fait attendre pendant plus d'un an; durant ce temps, un membre du SES a assuré l'intérim. 
( monitoring and evaluation», M\&E) ${ }^{61}$. En outre, il doit certifier et harmoniser les systèmes auprès des acteurs de la coopération belge au développement et évaluer la qualité de ces mêmes systèmes. Maintenant que le nouvel évaluateur spécial est nommé, cet arrêté royal du 25 avril 2014 va pouvoir être mis en application, ce qui pose un certain nombre de questions. Comment le SES va-t-il assurer ses nouvelles missions? Comment va-t-il certifier les systèmes de suivi et évaluation de l'ensemble des acteurs? Les acteurs de la coopération seront-ils tous certifiés de la même manière? Quel sera le processus? S'agira-t-il d'une certification annuelle ou d'une certification valable à plus long terme ? Etc.

Récemment, le SES a mené un long travail de consultation des acteurs, en vue d'établir sa programmation future. Cette consultation a eu pour effet d'inclure les différentes parties prenantes dans le cycle politique. Le SES a mis sur pied une étude pilote pour mener une réflexion sur la façon la plus adéquate de certifier et harmoniser les systèmes de suivi et évaluation des acteurs.

\subsubsection{La Société belge d'investissement pour les pays en développement (BIO-Invest)}

Particulièrement depuis ces dix dernières années, le rôle du secteur privé est considéré comme un facteur stratégique pour la promotion de la croissance économique et comme un outil important de lutte contre la pauvreté. La Société belge d'investissement pour les pays en développement (BIO-Invest) a ainsi été créée par une loi du 3 novembre $2001^{62}$. Elle vise à soutenir un secteur privé fort dans les pays en voie de développement et/ou émergents, et à permettre l'accès à une croissance durable ${ }^{63}$. Elle a un statut de société à part entière et distincte, pour lui permettre de réaliser son double objectif de rendement financier, d'une part, et de rendement dans le cadre du développement, d'autre part. Auparavant, c'était la CTB qui était chargée de cette compétence d'appui au secteur privé.

La création de BIO-Invest par l'État belge visait à répondre aux besoins de financement des PME, souvent peu soutenues par les institutions financières. Les PME sont financées à la condition de répondre à des critères de pertinence pour le développement, de respect des règles de la concurrence, de caractère additionnel et d'effet catalyseur. BIO-Invest a subdivisé ses activités de financement en trois domaines, à savoir les institutions financières, les entreprises et les infrastructures.

Dès sa création, BIO-Invest a proposé de réaliser des placements en vue d'optimiser la fiscalité des fonds investis. Alors que la démarche était admise sur le plan technicolégal, cela n'a pas été le cas sur le plan moral. BIO-Invest a connu un scandale éthique en 2012, lorsque les coupoles CNCD 11.11.11 (côté francophone) 11.11.11 (côté néerlandophone) ont dénoncé des placements de BIO-Invest dans des paradis fiscaux,

${ }^{61}$ Arrêté royal du 25 avril 2014 modifiant l'arrêté royal du 25 février 2010 portant création d'un service Évaluation spéciale de la coopération internationale, Moniteur belge, 22 mai 2014.

62 Loi du 3 novembre 2001 portant création de la Société belge d'investissement pour les pays en développement et modifiant la loi du 21 décembre 1998 portant création de la Coopération technique belge sous la forme d'une société de droit public, Moniteur belge, 17 novembre 2001.

63 BIO-Invest, « Rapport annuel », 2015. 
posant ainsi la question de l'utilisation de l'argent public dans des structures privées. Suite à ces événements, des mécanismes de contrôle plus poussé ont été mis en place.

\subsection{LA COOPÉRATION NON GOUVERNEMENTALE}

La coopération non gouvernementale est légalement définie comme « la coopération, financée ou cofinancée par la coopération belge au développement, dans laquelle un tiers, qui n'est pas un État étranger ni une organisation multilatérale, répond de l'exécution des interventions de coopération au développement, sur la base d'un système réglementaire de subvention ou d'une convention ${ }^{64}$.

Elle représente environ $22 \%$ du budget annuel de l'aide publique belge au développement, soit 246,3 millions d'euros en $2016^{65}$. Elle repose sur l'engagement de différents acteurs: principalement les ONG, mais aussi les établissements d'enseignement supérieur, les institutions scientifiques, les instituts de formation, les villes et communes, et les syndicats. Bien que la coopération au développement soit une compétence fédérale, la coopération non gouvernementale est linguistiquement structurée : chaque communauté possède ses acteurs.

\section{Graphique 1. Répartition du budget des acteurs non gouvernementaux} (en millions d'euros), 2016

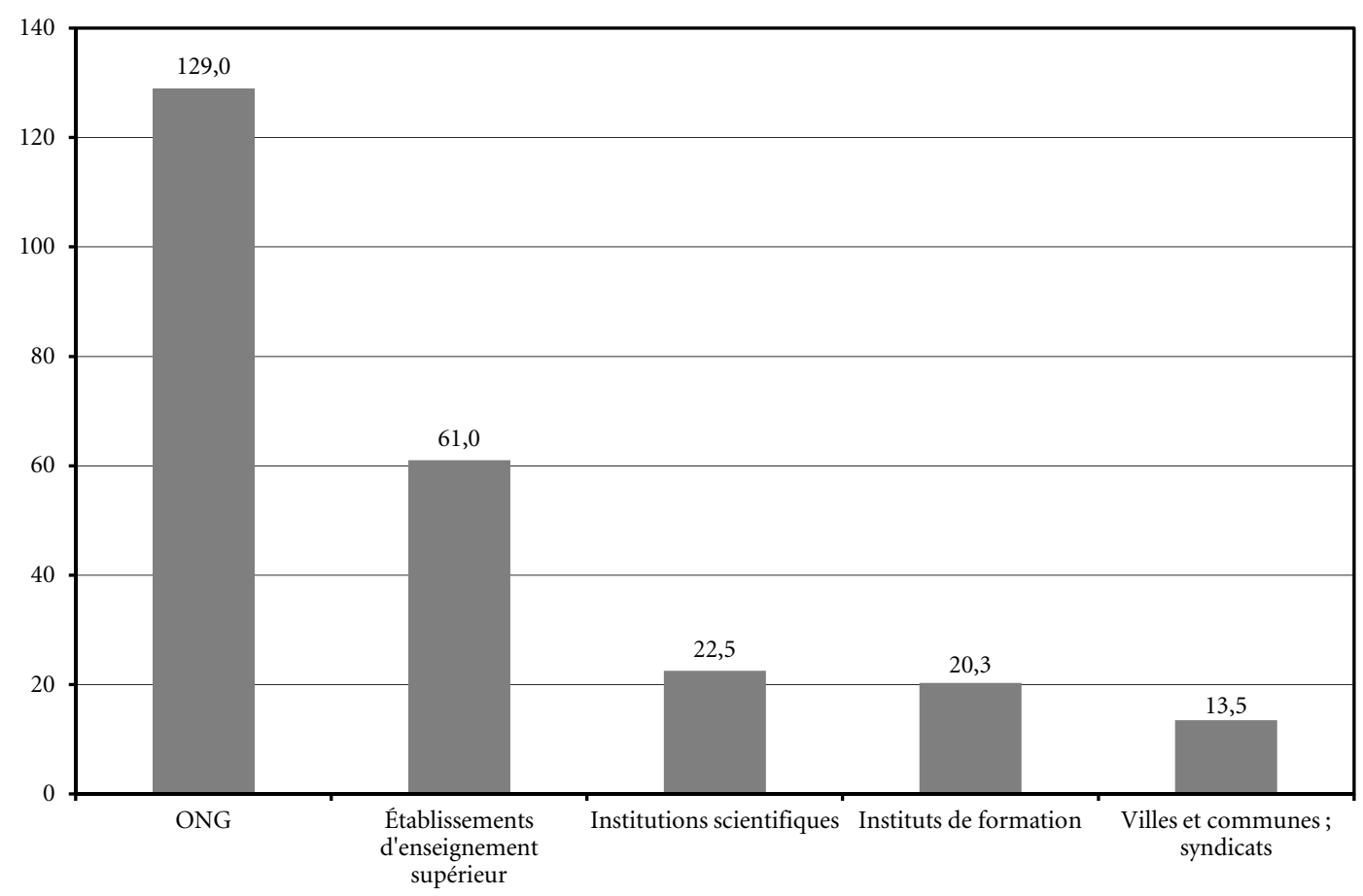

Source : APD belge, 2016.

${ }_{64}$ Loi du 19 mars 2013 relative à la coopération belge au développement, Moniteur belge, 12 avril 2013.

65 Chiffres de l'APD belge, 2016. 


\subsubsection{Les ONG de développement}

Ce point a pour ambition de dresser un état des lieux du secteur des ONG belges, entre la fin des années 1990 et le milieu de l'année $2017^{66}$. La légitimité sociale des ONG repose sur leurs contacts avec les acteurs locaux au Nord et au Sud. Nous allons voir ici la façon dont elles se sont transformées au cours des dernières années, ainsi que les relations qu'elles entretiennent aujourd'hui avec l'administration. Enfin, nous reviendrons sur un moment marquant de la réforme initiée par le ministre A. De Croo : un "screening" des capacités gestionnaires de toutes les ONG, effectué en 2016 en vue de l'attribution leurs subventions.

\section{Quelques préalables}

Les ONG existent en Belgique depuis de très nombreuses années (cf. infra), mais il a fallu attendre la loi du 25 mai 1999 relative à la coopération internationale belge ${ }^{67}$ pour voir émerger la notion d'agrément des ONG (devenue accréditation en septembre $2016^{68}$ ). Ce faisant, pour être reconnues, les ONG belges doivent avoir un statut d'asbl. Les principes de leur accréditation et de leur cofinancement sont réglés par les arrêtés royaux du 23 août $2015^{69}$ et du 11 septembre $2016^{70}$. Les ONG belges accréditées sont à ce jour un peu plus de septante. Elles mettent en œuvre des programmes de développement au Sud ou en Belgique pour un budget global de 129 millions d'euros annuels, soit plus de $50 \%$ du budget de la coopération non gouvernementale ${ }^{71}$. Il s'agit donc d'acteurs très importants dans la coopération belge indirecte.

Depuis la crise des années 1990, ce secteur particulier a connu plusieurs réformes politiques qui ont surtout porté sur des accords entre le gouvernement fédéral belge et le secteur des ONG, avec une modification de la rotation des engagements : les programmes sont passés à cinq ans et l'approche par projets a été supprimée. Le 4 mai 2009, le ministre de la Coopération au développement du gouvernement Van Rompuy (CD\&V/MR/PS/ Open VLD/CDH), Charles Michel (MR), conclut un accord-cadre avec le secteur des ONG en vue d'améliorer les collaborations avec l'administration. L'engagement du ministre porte sur l'augmentation de l'aide, l'amélioration de la cohérence des politiques, l'amélioration de l'efficacité de l'aide, l'accroissement de la collaboration entre les ONG et la DGD. Sur la base de cet accord-cadre, les ministres suivants en charge de la Coopération au développement, à savoir Paul Magnette (PS) et surtout Jean-Pascal Labille (PS), mettent en place les notions de concentration géographique (50 pays) et

66 Pour une analyse antérieure, cf. S. CHARNOvitz, "Les ONG : deux siècles et demi de mobilisation », op. cit. ; E. QUÉINNEC, « La croissance des ONG humanitaires », op. cit. ; G. STANGHERLIN, Les acteurs des ONG. L'engagement pour l'autre lointain, Paris, L'Harmattan, 2005 (cette publication s'est intéressée à l'évolution du secteur ONG belge, de la période postcoloniale jusqu'à la fin des années 1990).

67 Moniteur belge, $1^{\text {er }}$ juillet 1999.

68 Arrêté royal du 11 septembre 2016 concernant la coopération non gouvernementale, Moniteur belge, 29 septembre 2016.

69 Arrêté royal du 23 août 2015 modifiant l'arrêté royal du 25 avril 2014 concernant la subvention des acteurs de la coopération non gouvernementale, Moniteur belge, 31 août 2015.

70 Arrêté royal du 11 septembre 2016 concernant la coopération non gouvernementale, Moniteur belge, 29 septembre 2016.

71 Chiffres de l'APD belge, 2016. 
le principe de synergies à travers les "analyses contextuelles communes » des ONG (ACC). Enfin, Alexander De Croo (Open VLD) devient ministre de la Coopération au développement le 11 octobre 2014. Depuis lors, il mène une politique libérale et entreprend une réforme majeure, qui peut être considérée comme la plus importante après la réforme Moreels.

\section{Les principales caractéristiques}

Historiquement, les ONG de développement existent en Belgique depuis les années 1930. Depuis lors, le secteur s'est profondément transformé, passant d'ONG issues du milieu catholique à des ONG techniques (dans les années 1990) ${ }^{72}$. Aujourd'hui, le milieu catholique demeure toujours représenté dans le secteur des ONG, même s'il constitue moins une raison d'engagement qu'un réseau persistant.

Actuellement, il semblerait qu'une nouvelle génération d'ONG émerge, que nous qualifions de gestionnaire. Sous l'influence du nouveau paradigme de l'aide (efficacité, qualité, efficience, etc. : cf. supra), les ONG sont devenues plus complexes, soucieuses de leur performance et de leur bonne gestion au siège, notamment afin de démontrer ainsi, à l'administration et aux autres partenaires et financeurs, qu'elles sont des structures dignes de confiance.

Les ONG belges de développement mènent trois types d'action: l'éducation à la citoyenneté mondiale et solidaire (ECMS, nouvelle appellation de l'éducation au développement), l'action au Sud et le plaidoyer. En termes d'initiatives, au Sud, elles coordonnent aujourd'hui davantage les projets avec les acteurs locaux, en vue de renforcer les capacités de ces derniers. La plupart d'entre elles collaborent avec la région d'Afrique centrale pour des raisons historiques, ainsi qu'avec les quatorze pays prioritaires de la politique actuelle (cf. supra).

Face aux attentes de l'administration et du cabinet du ministre, qui exigent toujours davantage de professionnalisation, de rentabilité et de performance, et avec l'appui de leurs fédérations (Acodev et NGO Federatie, cf. infra), les ONG se sont engagées depuis plusieurs années dans des processus de reconnaissance de leur qualité (par exemple, en mobilisant des techniques d'évaluation de type Fondation européenne pour la gestion de la qualité - en anglais, European Foundation for Quality Management, EFQM ${ }^{73}$ ).

Le secteur des ONG belges est caractérisé par une multi-fragmentation. Les ONG sont réparties en fonction de leur taille (en termes de ressources humaines), de leur budget (qui est souvent lié à la taille: plus une ONG est grande en taille, plus ses moyens financiers sont importants) et de leur langue (ONG francophones, ONG néerlandophones et ONG bilingues).

72 Cf. S. CHARNOVITZ, « Les ONG : deux siècles et demi de mobilisation », op. cit.; E. QUÉINNEC, « La croissance des ONG humanitaires ", op. cit.

3 L'EFQM est un cadre de référence couramment employé en Europe dans un souci d'évaluation organisationnelle, qui met en évidence les points forts et les éléments à améliorer d'une organisation, tout en la comparant à des entités semblables dans une logique de benchmark. En bref, l'EFQM est basé sur la question suivante: "Qu'est-ce qui est décisif pour le succès d'organisations orientées vers l'excellence? ». Ces éléments ne sont ni des normes, ni des prescriptions, mais des indications sur les relations entre les actions de l'entreprise et les résultats. 
Les ONG francophones sont plus nombreuses et plus petites, et elles disposent de moyens financiers souvent moins importants que les ONG néerlandophones. Cela s'explique notamment par le fait qu'une partie des ONG néerlandophones ont fusionné il y a plusieurs années, renforçant ainsi leurs ressources humaines et leur budget. Par ailleurs, les ONG néerlandophones reçoivent davantage de financement en provenance d'entités fédérées et provinciales que les ONG francophones, et elles répondent davantage à des appels à propositions de l'Union européenne. Pour leur part, les ONG bilingues se divisent en deux sous-catégories. D’une part, les ONG belges : leur siège se situe généralement en région bruxelloise et elles sont souvent de plus grande taille que leurs sœurs francophones ou néerlandophones (par exemple, Action Damien). D’autre part, les ONG internationales : elles possèdent des succursales dans différentes régions du monde dont la Belgique (par exemple, Oxfam) et ont la particularité d'être de plus grande taille, avec un budget souvent plus important.

Les différences de moyens (en matière de ressources humaines et financières et de compétences internes) jouent en faveur des ONG néerlandophones et surtout internationales, qui peuvent répondre plus facilement aux attentes des bailleurs de fonds (DGD, Union européenne, etc.) en matière de bonne gestion. Or ces exigences se sont renforcées au cours des dernières années, en lien avec la Déclaration de Paris (cf. supra), modifiant de facto les conditions de concurrence entre ONG.

Les ONG n'échappent pas à la pilarisation de la société belge ${ }^{74}$. Les ONG du pilier chrétien sont les plus nombreuses. Cependant, il n'existe pas de lien de causalité marqué entre l'appartenance idéologique et les moyens humains et/ou financiers d'une ONG donnée.

En termes de moyens financiers, MSF Belgique - qui est par ailleurs une ONG humanitaire (et non de développement) - a clairement un statut particulier par rapport aux autres ONG belges. Ses revenus annuels s'approchent en effet des 350 millions d'euros, alors que ceux des autres ONG sont tout au plus d'une trentaine de millions d'euros ${ }^{75}$. Hormis MSF Belgique, les ONG belges de développement disposant des revenus les plus importants étaient, en 2014 et dans l'ordre décroissant : Oxfam, Caritas, Action Damien, UNICEF, Broederlijk Delen, Plan Belgique, Médecins du monde et Trias. Plusieurs d'entre elles sont donc des succursales d'ONG internationales: Oxfam, Caritas, UNICEF (qui est d'abord une agence d'exécution des Nations unies), Médecins du monde et Plan. Avec la coupole néerlandophone 11.11 .11 (cf. infra), ces ONG représentent près de $60 \%$ du budget total alloué aux ONG de développement en Belgique : environ 70 millions d'euros de subsides (sans compter MSF Belgique). Les $40 \%$ restants sont répartis entre toutes les autres ONG.

La question financière est primordiale pour le secteur, car les subsides fédéraux sont la première source de financement des ONG belges, créant un fort rapport de dépendance. Les fonds privés sont essentiellement obtenus par des campagnes de récolte, qui représentent au maximum $35 \%$ des ressources dont bénéficient les ONG belges. La

74 N. Molenaers, S. Dewachter, S. Dellepiane, « Moving into the New Aid Approach, Dilemmas for NGOs: The Belgian Case ", Public Administration and Development, volume 31, n 3, 2011, p. 188-204; N. Molenaers, L. Niss, H. HuYse, «The Belgian NGO Landscape and the Challenges of the New Aid Approach: Dealing with Fragmentation and Emerging Complexities », IOB Discussion Papers, Institute of Development Policy (IOB, Universiteit Antwerpen), n² 2, 2011.

75 Cf. «ONG Livre ouvert », 2014, www.ong-livreouvert.be. 
large proportion représentée par le financement public implique un affaiblissement de l'autonomie organisationnelle des ONG; or cette proportion a augmenté considérablement depuis les années $1980^{76}$. Cependant, dans les faits, les ONG peuvent toujours choisir d'intervenir dans un pays et/ou sur une thématique non prioritaire pour l'État belge.

Les données que nous avons récoltées relativement aux ONG belges de développement ${ }^{77}$ portent notamment sur la répartition entre fonds publics et fonds propres, le budget, l'affiliation linguistique, et la réussite ou non du screening de 2016.

Tableau 1. Corrélation entre les variables « Dépendance/indépendance aux fonds publics » et «Catégorie linguistique» (2016)

\begin{tabular}{|c|c|c|c|c|c|c|c|c|}
\hline & \multicolumn{7}{|c|}{ Dépendance/indépendance aux fonds publics } \\
\hline & & $\begin{array}{c}\text { ONG à } \\
\text { l'équilibre }\end{array}$ & $\begin{array}{c}\text { ONG } \\
\text { indépendantes }\end{array}$ & $\begin{array}{c}\text { ONG peu } \\
\text { dépendantes }\end{array}$ & $\begin{array}{c}\text { ONG } \\
\text { moyennement } \\
\text { dépendantes }\end{array}$ & $\begin{array}{l}\text { ONG très } \\
\text { dépendantes }\end{array}$ & $\begin{array}{c}\text { Données } \\
\text { non } \\
\text { disponibles }\end{array}$ & Total \\
\hline \multirow{5}{*}{ 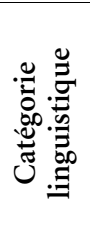 } & Francophones & 0 & 0 & 6 & 4 & 12 & 2 & 24 \\
\hline & Néerlandophones & 3 & 0 & 2 & 6 & 4 & 0 & 15 \\
\hline & Bilingues belges & 0 & 1 & 3 & 7 & 2 & 1 & 14 \\
\hline & \begin{tabular}{|l|} 
Bilingues \\
internationales \\
\end{tabular} & 2 & 4 & 3 & 1 & 4 & 1 & 15 \\
\hline & Total & 5 & 5 & 14 & 18 & 22 & 4 & 68 \\
\hline
\end{tabular}

Rem. : Le tableau ne porte que sur les ONG qui ont réussi le screening.

Le tableau 1 montre qu'il y a bien moins d'ONG indépendantes ou peu dépendantes des fonds publics que d'ONG moyennement ou très dépendantes de ceux-ci. Sans surprise, les ONG les plus indépendantes sont les ONG internationales. Dans la catégorie des ONG très dépendantes, figurent essentiellement des ONG francophones. Dans celle des ONG à l'équilibre, on ne trouve que des ONG internationales et des ONG néerlandophones.

Tableau 2. Corrélation entre les variables « Taille de l'ONG (en termes de budget) » et «Catégorie linguistique» (2016)

\begin{tabular}{|c|c|c|c|c|c|c|c|c|c|}
\hline & \multicolumn{8}{|c|}{ Taille de l'ONG (en termes de budget) } \\
\hline & & $\begin{array}{c}\text { XS } \\
\text { (moins de } \\
100000 \\
\text { euros) }\end{array}$ & $\begin{array}{c}S \\
\text { (moins de } \\
1000000 \\
\text { euros) }\end{array}$ & $\begin{array}{c}\mathrm{M} \\
\text { (moins de } \\
5000000 \\
\text { euros) }\end{array}$ & $\begin{array}{c}\mathrm{L} \\
\text { (moins de } \\
15000000 \\
\text { euros) }\end{array}$ & $\begin{array}{c}\mathrm{XL} \\
\text { (moins de } \\
30000000 \\
\text { euros) }\end{array}$ & $\begin{array}{c}\text { XXL } \\
\text { (plus de } \\
30000000 \\
\text { euros) }\end{array}$ & $\begin{array}{c}\text { Données } \\
\text { non } \\
\text { disponibles }\end{array}$ & Total \\
\hline \multirow{5}{*}{ 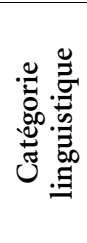 } & Francophones & 1 & 19 & 7 & 4 & 0 & 0 & 8 & 39 \\
\hline & Néerlandophones & 0 & 4 & 10 & 7 & 0 & 0 & 2 & 23 \\
\hline & Bilingues belges & 1 & 10 & 4 & 4 & 1 & 0 & 7 & 27 \\
\hline & \begin{tabular}{|l|} 
Bilingues \\
internationales
\end{tabular} & 0 & 1 & 1 & 8 & 3 & 1 & 1 & 15 \\
\hline & Total & 2 & 34 & 22 & 23 & 4 & 1 & 18 & 104 \\
\hline
\end{tabular}

Rem. : Les résultats présentés dans le tableau sont indépendants de la réussite du screening.

76 G. STANGHERLIN, Les acteurs des ONG, op. cit.

Ces données sont essentiellement issues de « ONG Livre ouvert », une base de données alimentée par les fédérations et les ONG, disponible sur le site Internet www.ong-livreouvert.be. Nous avons compilé ces données et mené des analyses de type tri croisé pour obtenir ces résultats. 
Relativement au budget annuel (obtenu par addition des subsides publics et des fonds propres), le tableau 2 montre que la catégorie $S$ (budget entre 100000 et 1000000 euros) est la plus représentée. Cette même catégorie est essentiellement constituée d'ONG francophones. Pour leur part, les ONG néerlandophones figurent surtout dans la catégorie M (budget entre 1000000 et 5000000 euros) et les ONG bilingues internationales dans la catégorie L (budget entre 5000000 et 15000000 euros). La catégorie XL (budget entre 15000000 et 30000000 euros) compte une ONG bilingue belge et trois ONG bilingues internationales, tandis que la catégorie XXL (budget de plus de 30000000 euros) n'accueille qu'une seule ONG, à savoir une ONG bilingue internationale.

\section{Les fédérations et coupoles}

Les fédérations et coupoles d'ONG constituent deux types de structures, qui tiennent des rôles différents et complémentaires. Les fédérations remplissent essentiellement un rôle de représentation de leurs membres auprès du politique, de traduction entre les demandes de l'administration et leurs ONG membres, ainsi que de renforcement des capacités de leurs ONG membres. Elles ont d'ailleurs été très impliquées à différents niveaux lors du screening, par exemple en traduisant les attentes de la DGD, en renforçant les capacités de leurs ONG membres à travers des formations, ou encore en faisant appel à des experts. Quant à elles, les coupoles défendent les intérêts des ONG face au monde politique et financent en partie des programmes grâce à des récoltes de fonds.

À l'origine, les fédérations et les coupoles d'ONG n'étaient pas linguistiquement séparées. Le processus de séparation sur une base communautaire a pris cours dans les années 1980-1990.

\section{Les fédérations}

Les années 1970 voient la création de deux fédérations nationales et bilingues, par regroupement de comités préexistants : d'une part, en 1970, Intercodev National, qui est une fédération nationale d'ONG d'envois (c'est-à-dire d'ONG qui dépêchent des coopérants dans les pays du Sud) ; d'autre part, en 1976, Coprogram National, qui est une fédération d'ONG de projets (en l'occurrence, principalement des ONG qui œuvrent dans le domaine de l'éducation au développement).

Ces fédérations se divisent ensuite par régime linguistique. En 1989, Coprogram National se scinde en deux entités distinctes : l'Association des ONG francophones (ADO) et Coprogram Vlaanderen. La scission d'Intercodev National a lieu en 1992 : elle donne naissance à Codef et à Intercodev Vlaanderen. En 1993, Coprogram Vlaanderen et Intercodev Vlaanderen fusionnent pour former Coprogram, qui est rebaptisée NGO Federatie en 2012. En 1997, ADO et Codef fusionnent pour former Acodev. De nos jours, Acodev est donc la fédération des ONG francophones et germanophones de coopération au développement, tandis que NGO Federatie est celle des ONG néerlandophones. Pour leur part, les ONG bilingues sont membres des deux fédérations.

Aujourd'hui, les fédérations sont au cœur du secteur, et ce pour plusieurs raisons. Tout d'abord, elles jouent le rôle de double traduction et de médiation du politique vers le secteur et inversement, puisqu'elles s'assurent que l'information soit transmise dans les deux sens. Ensuite, elles mettent en place une série de formations pour soutenir le 
secteur. Enfin, elles sont devenues un maillon dans la chaîne du financement des ONG, puisque celles-ci peuvent, via l'approche groupée, être financées par l'intermédiaire des fédérations (cf. infra), qui reçoivent d'ailleurs des budgets de la DGD pour assurer cette mission.

\section{Les coupoles}

Le Centre national de coopération au développement (CNCD, en néerlandais Nationaal Centrum voor Ontwikkelingssamenwerking - NCOS) est créé le 26 avril 1966, quatre collectifs d'associations de solidarité avec le Tiers-Monde ayant répondu à l'appel de la FAO pour éradiquer la faim dans le monde. Quelques mois plus tard, le CNCD organise la première "Opération 11.11.11 ${ }^{78}$ afin de récolter des fonds auprès de la population belge dans le but de financer des projets de développement dans le Tiers-Monde.

Les premières années sont marquées par une vision caritative mais, dans les années 1970, l'organisation évolue vers une coordination plus large d'une centaine d'associations membres. Le CNCD se fixe alors trois missions : financer des projets dans le Sud, interpeller le monde politique et informer le public belge. Par la suite, l'organisation s'intéresse aux enjeux mondiaux tels que la démocratie, la dette du Tiers-Monde, l'environnement, le renforcement de la société civile au Sud ou encore les droits humains.

En 1981, la demande d'autonomie de l'aile flamande aboutit à la scission en deux entités juridiques distinctes : le CNCD (aujourd'hui dénommé CNCD 11.11.11) et le NCOS (de nos jours, 11.11.11). En 1991, les deux coupoles sont formellement agréées en tant qu'ONG par le gouvernement, ce qui donne accès au cofinancement public. En 2003, les trois organisations syndicales (CSC, FGTB et CGSLB) rejoignent les coupoles, dont le nombre et la nature des organisations membres continuent de s'élargir.

En cinquante ans, les coupoles sont devenues des acteurs importants de la coopération belge au développement et sont considérées comme les représentantes des ONG et des autres membres du secteur indirect auprès du monde politique. Elles sont d'ailleurs présentes et proactives lors des négociations politiques qui concernent le secteur.

\section{Les ONG belges et la réforme actuelle}

L'actuel ministre de la Coopération au développement, Alexander De Croo (Open VLD), semble promouvoir depuis le début de son mandat une vision néo-libérale de la coopération au développement, plaçant le développement du secteur privé dans ses priorités politiques. Sa politique est en particulier incarnée par une nouvelle loi, datée du 16 juin $2016^{79}$, mobilisant notamment une approche orientée sur les résultats, des critères de qualité et un renforcement de la professionnalisation des « organisations de la société civile» (OSC, cf. infra). Sur le plan opérationnel, une restructuration importante

78 Les fondateurs du CNCD ont choisi le nom «11.11.11» pour rappeler qu'il n'y a pas de développement possible sans paix, et inversement, d'où le choix d'organiser cette récolte le $11^{\mathrm{e}}$ jour du $11^{\mathrm{e}}$ mois à la $11^{\mathrm{e}}$ heure, soit le moment où traditionnellement les anciens combattants fleurissaient les monuments aux morts.

79 Loi du 16 juin 2016 modifiant la loi du 19 mars 2013 relative à la coopération belge au développement, Moniteur belge, 30 juin 2016. 
de la coopération au développement a amené à une modification du statut de la CTB (devenue Enabel le $1^{\text {er }}$ janvier 2018, cf. supra).

Ce courant de réforme concerne également le secteur des ONG. Celles-ci doivent faire la preuve de la qualité de leur gestion et, partant, de leur capacité à bien gérer leurs programmes. Dans ce contexte, l'instrument politique qu'est le screening réalisé en 2016, et plus particulièrement les critères sur lesquels il a porté, est particulièrement intéressant à analyser dans un contexte de néo-managérialisation de la coopération au développement.

\section{Les évolutions du cadre légal}

Depuis l'adoption de la nouvelle loi, l'expression « organisation non gouvernementale » (ONG) est remplacée par « organisation de la société civile » (OSC) dans les textes officiels. Une OSC est définie par la loi du 16 juin 2016 comme «l'entité non étatique et sans but lucratif dans laquelle des gens s'organisent pour poursuivre des objectifs ou des idéaux communs ».

Quatre acteurs et une approche stratégique sont également ajoutés par la loi pour organiser l'ensemble des acteurs non gouvernementaux. Primo, l'acteur institutionnel (AI) c'est-à-dire l'organisation qui est fondée par un pouvoir public ou qui est contrôlée ou gérée directement ou indirectement par lui (cf. infra). Secundo, la fédération, qui est l'organisation qui représente l'ensemble ou une partie des organisations accréditées et qui joue le rôle d'interface entre l'administration et ces organisations accréditées. Tertio, la coupole, qui est l'organisation de la société civile dont les membres sont d'autres organisations de la société civile actives dans le domaine de la coopération au développement. Quarto, l'organisation accréditée, qui est l'organisation qui a le droit de solliciter une subvention dans le cadre de la coopération non gouvernementale. Quinto, le cadre stratégique commun (CSC), qui est l'ensemble des choix stratégiques effectués conjointement par les organisations accréditées dans un pays ou sur un thème, à partir d'une analyse contextuelle.

Pour être accréditée, l'organisation doit satisfaire aux conditions spécifiques suivantes, fixées par la loi : être constituée en asbl, avoir pour objet social un ou plusieurs objectifs de la coopération belge au développement, avoir un chiffre d'affaires annuel de minimum 50000 euros, avoir des ressources humaines suffisantes (à savoir au moins deux équivalents temps plein), être autonome (c'est-à-dire n'avoir aucun membre de son conseil d'administration qui fasse partie du SPF Affaires étrangères, Commerce extérieur et Coopération au développement, d'Enabel, de BIO-Invest ou de la cellule stratégique du ministre) et avoir réussi le "screening» (cf. infra).

Pour être éligible au financement de la DGD (pour une durée de 5 ans), tout programme doit par ailleurs ${ }^{80}$ : être cohérent avec le plan stratégique établi par le ou les demandeur(s), indiquer par résultats ou groupes de résultats de quelle manière il contribuera à la réalisation d'un ou plusieurs cadres stratégiques communs, présenter une approche orientée vers les résultats (c'est-à-dire orientée par une théorie du changement, avec une description des outcomes attendus, des indicateurs quantitatifs et qualitatifs, et des indicateurs spécifiques, mesurables, réalisables et pertinents), avoir réalisé une analyse

Arrêté royal du 11 septembre 2016 concernant la coopération non gouvernementale, Moniteur belge, 20 septembre 2016. 
des risques, respecter les critères de qualité, ne pas dépasser la durée maximale fixée de 5 ans, présenter un budget précis et au moins égal au montant fixé (à savoir supérieur ou égal à 2000000 euros par an pour un programme Sud, et supérieur ou égal à 750000 euros par an pour un programme d'ECMS), concentrer son budget dans un ou plusieurs cadres stratégiques communs.

Un lien direct peut être établi entre la managérialisation et le processus de financement des structures. Si les OSC rendent un programme suffisamment conséquent budgétairement, elles sont éligibles à un financement direct. $\mathrm{Si}$, au contraire, elles rendent un programme inférieur à la somme fixée, elles ont deux possibilités : soit elles concluent un partenariat avec une ou plusieurs structures pour remettre un programme commun ( "demande groupée ») et suffisamment important pour atteindre le niveau de budget requis, soit elles postulent pour un financement indirect (c'est-à-dire géré par les fédérations d'ONG).

L'accessibilité aux subsides publics démontre la managérialisation des ONG. En effet, pour répondre aux exigences du bailleur (ici, l'État belge), les ONG doivent être performantes, être de grande taille et a priori être dans une situation d'importante capacité de gestion. Quant aux entités de petite taille, elles peuvent se rassembler en déposant une demande groupée avec le soutien de leur fédération, diminuant potentiellement la gestion et le suivi administratif pour la DGD et répondant ainsi à une nécessité de rationalisation mais aussi à un souci de décentralisation, puisque le financement de ces projets est pratiqué indirectement (par les fédérations). Ce dernier point pose la question du positionnement des fédérations d'ONG, qui, en assurant un rôle d'intermédiation pour les petites ONG, deviennent juges et parties, remettant possiblement en question leur rôle de structure représentative du secteur vis-à-vis des responsables politiques.

\section{L'opération d'évaluation des ONG : le «screening "}

Durant l'année 2016, les ONG belges de développement ${ }^{81}$ ont été soumises à une analyse approfondie de leurs performances en matière de gestion - un screening - basée sur une dizaine de domaines de gestion définis par un cabinet de consultance privé (BDO) et mis en œuvre par un autre cabinet de consultance indépendant (Deloitte) ${ }^{82}$. Ces domaines de gestion sont : la gestion financière, la gestion stratégique, la gestion des processus, la gestion par résultats, la gestion des partenariats, la gestion des risques, la gestion $\mathrm{du}$ personnel, la prise en compte des thèmes transversaux, la transparence, la complexité (composée de la taille, la dispersion géographique, la dispersion thématique, la diversité des partenaires, la dispersion des donneurs d'ordre, le volume des moyens humains). La réussite du screening a déterminé l'accès aux subsides publics. Il s'est dès lors agi d'un enjeu majeur pour le secteur.

Ce screening a pris la forme d'un audit externalisé par la DGD, car cette dernière n'est pas en mesure d'effectuer une vérification d'une telle ampleur en raison de la réduction de son personnel opérée depuis quelques années. Par ailleurs, cet examen a porté

81 Bien que les autres acteurs de la coopération indirecte aient été également soumis au screening, il ne sera question ici que des ONG.

82 Il est à noter que ce n'est pas la première fois qu'il est ainsi procédé à un screening. Un exercice semblable avait déjà été réalisé en 2006 (par le cabinet de consultance PriveWaterhouseCoopers - PWC), mais il n'avait pas eu pour conséquence une réduction du nombre des ONG ayant accès aux subsides publics. 
essentiellement, voire uniquement, sur les processus. L'évaluation a été basée sur deux variables : la qualité du système de gestion et la complexité des activités ou des thématiques couvertes (y compris leur dispersion géographique) ${ }^{83}$. Concernant ces dimensions, les ONG ont reçu une note allant de $\mathrm{A}$ à $\mathrm{D}$, la note $\mathrm{D}$ étant une note d'exclusion. Pour obtenir un bon classement (à savoir une note $\mathrm{A}, \mathrm{B}$ ou $\mathrm{C}$ ) les $\mathrm{ONG}$ devaient, idéalement, présenter un degré de complexité peu élevé et une gestion des risques optimale. Néanmoins, une marge de manœuvre était tolérée. Par exemple, les structures présentant un niveau de complexité élevé parce qu'elles travaillent dans de nombreux secteurs ou pays ont pu contrebalancer cela par un niveau de gestion maîtrisé.

L'approche méthodologique proposée par l'équipe de consultants, pour la réalisation du screening ainsi que pour la définition des critères eux-mêmes, a fait l'objet de nombreuses critiques de la part des ONG. En effet, l'approche choisie ne permet pas de vérifier si la structure considérée dispose effectivement d'un système de gestion efficace, puisqu'elle repose uniquement sur l'analyse de documents papier, sans observation des pratiques des ONG. Les ONG ont donc considéré que cette approche n'était pas représentative de leur travail. Elles l'ont même surnommée le «screening oui-non », car soit l'ONG disposait du document (par exemple, une charte sur le genre), soit elle n'en disposait pas. En outre, il n'y a pas eu de vérification quant à l'application effective ou non, sur le terrain, des principes présentés dans les documents. Les ONG ont également questionné la mise en œuvre du screening. Les structures ont dû produire un grand nombre de documents, parfois inexistants, pour répondre aux exigences du bailleur dans un temps imparti extrêmement court. Ce délai n'a pas permis aux consultants une analyse organisationnelle en profondeur, surtout relativement à un domaine dans lequel ils n'avaient pas beaucoup d'expérience.

En ce qui concerne le cadre légal, le ministre A. De Croo a indiqué que le processus avait été discuté avec les fédérations d'ONG ${ }^{84}$. Quant à la DGD, elle est volontairement restée muette durant tout le processus afin, a-t-elle déclaré, de garantir une impartialité de traitement entre les structures et de se prémunir de toute critique éventuelle.

Les résultats détaillés du screening n'ont pas été rendus publics. Toutefois, selon les informations récoltées sur le terrain, environ un dixième des ONG ont arrêté le processus en cours de route, et à peu près deux dixièmes supplémentaires ont échoué. In fine, l'exercice a donc exclu un tiers des acteurs. Deux ONG ont introduit un recours devant le Conseil d'État (institution devant laquelle toute personne morale peut contester un acte administratif irrégulier qui lui aurait causé un préjudice) ; l'affaire est actuellement en cours.

83 Plus une ONG travaille sur différentes thématiques et dans différents pays, plus elle est considérée comme complexe.

Chambre des représentants, Commission des Relations extérieures, Compte rendu intégral, $\mathrm{n}^{\circ}$ 437, 7 juin 2016. 
Tableau 3. Proportion d'ONG ayant échoué au screening de 2016 ou ayant arrêté en cours de processus

\begin{tabular}{|l|c|c|c|}
\hline Catégorie linguistique & Nombre total d'ONG & $\begin{array}{c}\text { Nombre d'ONG ayant } \\
\text { échoué ou abandonné }\end{array}$ & $\begin{array}{c}\text { Pourcentage d'échecs } \\
\text { ou d'abandons }\end{array}$ \\
\hline ONG francophones & 39 & 15 & $38,5 \%$ \\
\hline ONG néerlandophones & 23 & 8 & $34,8 \%$ \\
\hline ONG bilingues & 42 & 13 & $31,0 \%$ \\
\hline Total & 104 & 36 & $34,6 \%$ \\
\hline
\end{tabular}

Source : «ONG Livre ouvert », 2016, www.ong-livreouvert.be.

Les ONG francophones sont 38,5\% à avoir échoué ou abandonné, contre 34,8 \% des ONG néerlandophones et 31,0 \% des ONG bilingues (cf. Tableau 3). Cela s'explique, en partie, par le fait que les ONG francophones sont de plus petite taille que leurs consœurs néerlandophones et bilingues. Les ONG bilingues ayant échoué ou abandonné sont uniquement des ONG belges ou des petites ONG internationales; aucune des grandes ONG internationales n'a raté ou abandonné.

De nouveaux enjeux émergent dès lors. Les ONG les plus compétitives en matière de gestion sont favorisées par ce type de processus managérial, générant ainsi un rapport de concurrence entre $\mathrm{ONG}^{85}$. Deux profils d'ONG se distinguent. Le premier est l'ONG de grande taille qui dispose, a priori, de moyens humains et financiers conséquents, et peut donc se permettre d'engager des personnes - gestionnaires - qui sont spécifiquement en charge de ce type d'exercice. Le second est l'ONG internationale qui, en plus de disposer de moyens financiers et humains importants, bénéficie aussi de compétences en interne. Par exemple, si la GAR est une technique de gestion qui fait son apparition depuis quelques années en Belgique, elle est mise en œuvre depuis de nombreuses années dans d'autres pays, comme le Canada ou le Royaume-Uni. Ainsi, la succursale belge d'une ONG internationale peut faire appel à son homologue canadienne ou britannique pour être formée et pour traduire les processus dans sa propre structure. Dans ce contexte, ce sont par conséquent les ONG de grande taille et internationales qui sont les plus susceptibles d'être compétitives face aux nouveaux modes de financement et de gestion attendus par l'administration et le monde politique.

En réponse à ces enjeux et compte tenu du contexte néo-managérial général, les ONG belges de développement sont soumises à une nouvelle contrainte pour démontrer leur légitimité. Si celle-ci était à l'origine essentiellement basée sur leur assise sociétale et sur leur connaissance du terrain au Sud, aujourd'hui ces ONG doivent faire preuve d'une légitimité nouvelle, par leur bonne gestion et leur performance.

Parallèlement à ce processus d'analyse de leurs compétences gestionnaires, les ONG travaillent désormais dans une logique de travail en partenariat: elles ont élaboré des cadres stratégiques communs (CSC) ${ }^{86}$, sous la forme de 33 groupes de travail inter-ONG, pour favoriser la concentration et la cohérence des acteurs indirects de la coopération belge. La loi du 16 juin 2016 dispose à cet égard que «le CSC sert de référence pour

85 S. COUPRIE, «Le management stratégique des ONG ou la quête de légitimité », Mondes en développement, $\mathrm{n}^{\circ} 159,2012$, p. 59-72.

86 Rappelons que les CSC ont été précédés par les analyses contextuelles communes (ACC) ; celles-ci avaient été instaurées par le ministre J.-P. Labille, qui a initié le projet de synergie entre les acteurs indirects (cf. supra). 
l'élaboration des programmes de ces organisations, en ce compris l'identification et la mise en œuvre des synergies et complémentarités entre elles ; il constitue la base sur laquelle des enseignements sont tirés et partagés, et sur laquelle un dialogue stratégique est mené avec l'administration et les autres acteurs de la coopération belge au développement. (...) Le CSC constitue le cadre général de référence pour les programmes ».

Il est à noter que ces CSC ont constitué une stratégie du secteur des ONG pour contourner la logique initialement suggérée par le ministre. En effet, loin de s'être vu imposer les CSC, les ONG ont proposé ceux-ci au ministre afin de répondre à ses demandes stratégiques tout en contrant son idée de confier à Enabel le monopole du financement des programmes de la coopération belge via des appels à proposition («call for proposal ») - ce qui était inacceptable pour les ONG en termes d'autonomie et d'indépendance. Les CSC ont été globalement bien reçus par le secteur, qui a reconnu l'intérêt de cette démarche coopérative, même si aucune unanimité ne règne : certains considèrent que les CSC sont une opportunité de tirer des enseignements, alors que d'autres estiment qu'il s'agit d'une simple perte de temps.

\subsubsection{Les acteurs hors ONG}

Outre les ONG, divers acteurs non gouvernementaux sont actifs dans la coopération belge : les établissements d'enseignement supérieur, les institutions scientifiques, les instituts de formation, les villes et communes, et les syndicats.

\section{Les établissements d'enseignement supérieur}

La « coopération universitaire » est régie par six conventions conclues entre l'État belge, les établissements d'enseignement supérieur francophones (universités et hautes écoles), représentés par la Commission de la coopération au développement de l'Académie de recherche et d'enseignement supérieur (ARES-CCD, anciennement Commission universitaire pour le développement du Conseil interuniversitaire de la Communauté française - CIUF-CUD), et les établissements d'enseignement supérieur flamands, représentés par le Bureau Universitaire Ontwikkelingssamenwerking du Vlaamse Interuniversitaire Raad (VLIR-UOS). Ces conventions s'accompagnent de plans quinquennaux, qui déterminent les objectifs et les moyens prévus pour ce type de coopération.

L'ARES-CCD et le VLIR-UOS soutiennent des partenariats entre les établissements d'enseignement supérieur belges et ceux des pays du Sud, pour les renforcer dans leurs missions de formation, de recherche et de service à la société, ainsi que dans leur rôle d'acteur du développement. Elles ont donc un rôle de relais entre les différentes universités et hautes écoles belges et leurs homologues des pays partenaires. Les universités appuient également des projets de recherche en soutien à la politique, notamment à travers les projets de type ACROPOLIS. 
L'ARES-CCD dispose d'un budget annuel de 28,04 millions d'euros pour ce poste et le VLIR-UOS d'un budget de 32,75 millions d'euros ${ }^{87}$. Il est important de noter que, à elle seule, la coopération universitaire dispose de près de $30 \%$ du budget de la coopération non gouvernementale. Elle constitue donc le deuxième acteur de la coopération non gouvernementale après les ONG.

\section{Les institutions scientifiques}

Les institutions scientifiques concernées ici sont l'Institut de médecine tropicale (IMT, Anvers), le Musée royal de l'Afrique centrale (MRAC, Tervuren) et l'Institut royal des sciences naturelles de Belgique (IRSNB, Bruxelles).

L'IMT est financé à hauteur de 15 millions d'euros par an par la coopération belge au développement, ce qui représente environ $24 \%$ du budget annuel de l'IMT. Il développe et/ou entretient des partenariats avec des laboratoires, des instituts et des programmes de recherche de par le monde. Il contribue également sur le terrain dans le secteur de la santé.

La DGD est le principal partenaire financier du MRAC pour ses activités de coopération au développement. Le cadre de référence de ces activités se trouve dans un protocole de collaboration signé entre le SPF Politique scientifique fédérale et le SPF Affaires étrangères, Commerce extérieur et Coopération au développement. Le soutien de la coopération belge au développement permet au MRAC de contribuer à la diffusion des connaissances en Afrique auprès d'un large public et du monde scientifique. En aidant des institutions africaines dans leur mission et dans leur recherche commune, le MRAC contribue au développement durable de l'Afrique.

L'IRSNB œuvre dans différents domaines : la recherche scientifique en sciences naturelles, l'expertise scientifique au service des autorités publiques, la conservation et la gestion des collections patrimoniales scientifiques, la diffusion de la connaissance scientifique auprès du grand public.

\section{Les instituts de formation}

L'Association pour la promotion de l'éducation et de la formation à l'étranger (APEFE) et la Vlaamse Vereniging voor Ontwikkelingssamenwerking en Technische Bijstand (VVOB) ont pour objectif global la lutte contre la pauvreté par le renforcement (institutionnel, technique, administratif et de gestion) d'institutions étatiques, paraétatiques ou multilatérales localisées dans un pays en voie de développement à travers la formation. L'État belge subventionne leurs programmes triennaux d'activités sur la base d'une convention générale datant du 9 avril 2008. Chaque programme triennal fixe les objectifs à atteindre par pays, les résultats à produire et les moyens à mettre en œuvre.

L'APEFE dispose d'un budget annuel d'environ 9,5 millions d'euros. Quant à elle, la VVOB dispose d'un budget total d'environ 11,92 millions d'euros par an : 10,72 millions d'euros

${ }^{87}$ Chiffres de l'APD belge, 2016. 
provenant de la DGD ${ }^{88}$, près de 300000 euros versés par le gouvernement flamand, et près de 900000 euros issus de dons.

\section{Les villes et communes}

Les villes et communes viennent en appui aux communes du Sud dans les pays prioritaires définis par la Belgique. Leurs associations respectives ont pour mission de soutenir les initiatives des communes wallonnes (Union des villes et communes de Wallonie UVCW), bruxelloises (Association de la ville et des communes de la Région de BruxellesCapitale - AVCB) et flamandes (Vereniging van Vlaamse Steden en Gemeenten - VVSG) dans leurs projets de coopération. Ces structures mènent leurs actions au niveau local.

L'UVCW dispose d'un budget d'environ 1,7 million d'euros par an pour ce poste, et l'AVCB d'un budget de 1,1 million d'euros. Les deux organes collaborent structurellement. Pour sa part, la VVSG reçoit 520000 euros du gouvernement flamand pour une durée de trois ans et 1,2 million d'euros par an du niveau fédéral (soit au total environ 1,4 million d'euros par an) ${ }^{89}$.

\section{Les confédérations syndicales}

Depuis 2002, la coopération belge soutient les programmes de coopération syndicale initiés par l'Institut d'éducation ouvrière internationale (IEOI) de la Confédération des syndicats chrétiens (CSC), par l'Institut de formation syndicale internationale (IFSI) de la Fédération générale du travail de Belgique (FGTB) et par le Mouvement pour la solidarité internationale (MSI) de la Centrale générale des syndicats libéraux de Belgique (CGSLB).

Une convention datant du 6 juin 2007 et signée par les trois confédérations syndicales belges fixe le champ d'action de cette coopération. Elle vise principalement le renforcement des organisations syndicales sur le plan du développement institutionnel, de l'indépendance, de la démocratie, et dans le but de faire progresser la justice sociale.

L'IEOI, l'IFSI et le MSI ont aussi pour objectif de renforcer le dialogue social entre les pouvoirs publics, le patronat et les syndicats locaux dans les pays du Sud. Les trois structures sensibilisent aussi leurs membres aux questions de coopération au développement. Enfin, elles collaborent étroitement avec les ONG de la famille idéologique à laquelle elles sont historiquement liées : l'IEOI avec les ONG Solidarité mondiale et Wereld Solidariteit, qui sont les ONG de développement du Mouvement ouvrier chrétien (MOC) et de beweging.net et de leurs organisations membres, et l'IFSI avec les ONG Solidarité socialiste (Solsoc) et Fonds voor Ontwikkelingssamenwerking Socialistische Solidariteit (FOS) ${ }^{90}$. Ces collaborations sont désormais renforcées par la logique de partenariat promue par la réforme actuelle (cf. supra). En termes de budget, chacun de ces instituts syndicaux reçoit environ 1,2 million d'euros par an de subsides fédéraux. Ils sont aussi soutenus financièrement par leur famille syndicale.

88 Chiffres de l'APD belge, 2016.

${ }_{90}$ Chiffres de 2015.

90 En l'absence d'ONG de la famille libérale, le MSI collabore avec Solidarité mondiale et Wereld Solidariteit. 


\subsubsection{Mise en perspective}

Les acteurs non gouvernementaux belges sont variés, tant en termes d'origines que de pratiques, et ils se partagent un peu moins de la moitié du budget de la coopération non gouvernementale. Dans le cadre de la dernière réforme, ces acteurs viennent d'être regroupés sous le nom d' «acteurs de la coopération non gouvernementale » (ACNG), structurant le canal non gouvernemental en deux grands types d'acteurs : les organisations de la société civile (OSC, cf. supra) et les acteurs institutionnels (AI).

Les AI sont désormais représentés par la Fédération des acteurs institutionnels belges $\left(\right.$ FIABEL) ${ }^{91}$, qui regroupe neuf organisations : l'ARES-CCD et le VLIR-UOS, l'IMT, l'APEFE, la VVOB, les trois associations régionales de villes et communes, et l'asbl Africalia (créée en 2000 à l'initiative du secrétaire d'État E. Boutmans). Cette nouvelle fédération est chargée du dialogue et de la défense des intérêts communs de ses membres à l'égard des autorités fédérales, de la professionnalisation plus poussée de ses membres, de la promotion de la complémentarité, la synergie et l'apprentissage conjoint au sein du secteur. Elle collabore intensivement en particulier avec Acodev et NGO Federatie, qui mettent aussi leur expertise technique à la disposition des AI.

Quant aux syndicats, ils font partie de la catégorie OSC, au même titre que les ONG. Cela s'explique par le fait qu'ils ont des connexions et des actions directes avec les ONG de leur famille idéologique, et qu'ils sont membres des coupoles depuis 2003.

\subsection{LES ACTEURS PÉRIPHÉRIQUES}

Les acteurs dits périphériques ont eux aussi évolué avec le temps.

\subsubsection{La Belgian First Aid and Support Team (B-FAST)}

La Belgian First Aid and Support Team (B-FAST) a été créée en $2003^{92}$, en réponse à la nécessité de mieux coordonner les actions de l'État belge en cas d'aide d'urgence ${ }^{93}$. Près de $15 \%$ du budget annuel de la coopération lui sont consacrés. B-FAST dispose également de financements du Ministère de la Défense, du SPF Intérieur, du SPF Santé publique, Sécurité de la chaîne alimentaire et Environnement, et du SPF Affaires étrangères, Commerce extérieur et Coopération au développement.

91 La FIABEL est une association de fait, donc sans statuts déposés. Elle est hébergée au VVOB.

92 Cf. T. CoOsEmAns, «B-FAST et l'aide d'urgence à l'étranger », Courrier hebdomadaire, CRISP, n²277, 2015.

93 L'aide d'urgence est légalement définie comme «l'action consistant en tout envoi à l'étranger de moyens humains et/ou matériels, à évaluer au cas par cas en fonction de la spécificité de la situation du pays touché par la catastrophe ou la calamité » (à l'exclusion de l'aide d'urgence en matière de suivi et de réhabilitation, telle que définie dans l'arrêté royal du 19 novembre 1996 relatif à l'aide d'urgence et de réhabilitation à court terme en faveur des pays en voie de développement). Cette aide d'urgence est limitée à dix jours, sauf décision contraire du conseil de coordination. Cf. ibidem. 
Pour que B-FAST intervienne, une série d'éléments doivent être rassemblés : le désastre doit être d'ampleur, le pays sinistré doit avoir adressé une demande d'intervention à la communauté internationale, et il ne doit pas y avoir de conflit armé dans la zone d'intervention. Le Conseil des ministres est l'organe de décision. Outre un conseil de coordination (qui se réunit au moins deux fois par an), B-FAST se compose d'un comité de planification (chargé d'apporter un soutien stratégique au secrétariat) et d'un comité consultatif (qui soumet des avis techniques et d'amélioration du dispositif). Les interventions de B-FAST sont de cinq types : recherches et sauvetage de personnes; purification d'eau ; mise en place d'abris d'urgence temporaires ; installation d'hôpitaux de campagne ; gestion d'inondations.

\subsubsection{Le Fonds belge pour la sécurité alimentaire (FBSA)}

Jusqu'à sa fermeture en septembre 2015, le FBSA était un instrument unique de financement, axé sur l'amélioration de la sécurité alimentaire des populations vulnérables situées dans les zones de l'Afrique subsaharienne et présentant un grand risque d'insécurité alimentaire. Résultant d'une initiative parlementaire, il était alimenté par des fonds de la Loterie nationale.

La gestion du FBSA relevait du ministre fédéral en charge de la Coopération au développement, qui en assumait également la responsabilité. Le suivi du FBSA était assuré par le Groupe de travail spécial FBSA, composé de représentants de chaque groupe parlementaire, du ministre et de représentants de ses services (DGD), de représentants des ONG belges, de la CTB et des organisations multilatérales partenaires du FBSA.

Au cours de son histoire, le FBSA a connu quatre évolutions majeures. Entre 1989 et 1998, il est appelé le Fonds de survie pour le Tiers-Monde et dispose d'un budget de 10 milliards de francs belges (environ 250 millions d'euros) pour une durée de 15 ans, financé par la Loterie nationale. Entre 1999 et 2009, il porte le nom de Fonds belge de survie et dispose d'un budget de 10 milliards de francs belges pour une durée de 10 ans, versé par la Loterie nationale. À partir de 2008, une réflexion interne est initiée à la suite d'une évaluation. Par une loi du 19 janvier $2010^{94}$, il devient le FBSA et dispose d'un budget de 250 millions d'euros pour la période $2010-2017^{95}$. Ses principales évolutions se situent dans son approche, qui est devenue une approche par programme, davantage intégrée, multi-acteurs et multisectorielle. Enfin, le FBSA est supprimé en 2015 par le gouvernement fédéral Michel ; désormais, la politique de soutien à la sécurité alimentaire est uniquement gérée par la DGD.

94 Loi du 19 janvier 2010 abrogeant la loi du 9 février 1999 portant création du Fonds belge de survie et créant un Fonds belge pour la sécurité alimentaire, Moniteur belge, 11 février 2010.

5 Actuellement, le FBSA n'est plus doté de nouveaux financements, mais les programmes en cours pourront être menés à terme. 


\section{CONCLUSION}

Le présent Courrier hebdomadaire est tout d'abord revenu sur des éléments contextuels historiques, fondateurs, nationaux et internationaux, qui ont marqué l'histoire de la coopération belge au développement. En près de 60 ans, une relation coloniale fait place à une relation de partenariat relativement réciproque. En Belgique, un moment majeur de ce cheminement est la crise de la fin des années 1990. En effet, c'est à cette époque qu'est fondée la coopération belge au développement sur le plan institutionnel, telle que l'on la connaît aujourd'hui. Le cycle politique est ensuite fragmenté entre la programmation politique (prise en charge par l'administration), l'opérationnel (assuré par la CTB, aujourd'hui Enabel) et l'évaluation (effectuée par le SES). Cette période de crise questionne également le statut des ONG, mais les acteurs de l'époque s'accordent sur la légitimité sociétale de celles-ci. Il s'est donc véritablement agi d'une période charnière, car les ONG sont alors devenues officiellement parties prenantes dans les débats politiques relatifs à la coopération, et se sont vues dotées d'un statut face à l'administration à travers le processus d'agrément. Le rôle propre que l'État a alors donné aux ONG a été déterminant dans tous les processus de négociations qui ont suivi.

Ensuite, l'étude a montré la place prépondérante qu'ont eue les institutions internationales dans le façonnage de l'idéologie de la coopération belge au développement. En l'occurrence, ce ne sont pas toujours les mêmes instances qui ont donné le ton, les Nations unies, les agences de Bretton Woods (BM et FMI) ou encore l'OCDE se partageant le leadership idéologique en fonction des périodes. Cet article a aussi souligné, d'une part, l'émergence de l'idéologie dominante actuelle, cristallisée dans la Déclaration de Paris (2005) et sa volonté de mener une aide plus efficace et, d'autre part, les nombreux accords qui ont suivi entre les pays donateurs et receveurs en vue de renforcer et améliorer les accords de Paris.

Enfin, ce Courrier hebdomadaire a passé en revue le grand nombre d'acteurs de la coopération belge au développement. La coopération multilatérale, importante en termes de budget, connaît une stabilité dans le temps et tend à se renforcer. La coopération bilatérale est avant tout constituée d'Enabel. Née d'une crise, cette instance est devenue en quelques années une actrice résolument incontournable, position qui semble se consolider encore avec son récent passage au statut d'agence, et diffuse aujourd'hui une vision entrepreneuriale de la coopération. La coopération non gouvernementale est quant à elle composée d'acteurs extrêmement variés, donnant l'impression d'un troisième pilier quelque peu « fourre-tout »(ONG et établissements d'enseignement supérieur, mais aussi institutions scientifiques, instituts de formation, villes et communes, et syndicats). Elle tend toutefois vers une certaine cohérence suite à la récente catégorisation entre organisations de la société civile (OSC, dont les ONG), d'une part, et les acteurs 
institutionnels (AI, représentés par la FIABEL, nouvellement créée), d'autre part. Plus spécifiquement en ce qui concerne les ONG, nous avons fait état des récentes évolutions, orientées vers la performance, l'efficacité ou encore les synergies. En particulier, les ONG se voient désormais dans l'obligation de démontrer leur bonne gestion si elles veulent survivre.

Ce faisant, aujourd'hui, tout porte à croire que nous sommes en présence d'une nouvelle génération d'ONG, que nous qualifions de gestionnaire. Cette génération se construit sur un mode de management différent, qui non seulement suppose de faire appel à de nouveaux profils de travailleurs davantage orientés vers la gestion, le marketing ou la communication, mais qui aussi transforme profondément le secteur en tant que tel. 


\section{CENTRE DE RECHERCHE ET D'INFORMATION SOCIO-POLITIQUES}

Le CRISP, Centre de recherche et d'information socio-politiques, est un organisme indépendant. Ses travaux s'attachent à montrer les enjeux de la décision politique, à expliquer les mécanismes par lesquels elle s'opère, et à analyser le rôle des acteurs qui y prennent part, que ces acteurs soient politiques, économiques, sociaux, associatifs, etc.

Par ses publications, le CRISP met à la disposition d'un public désireux de comprendre la société belge des informations de haute qualité, dans un souci d'exactitude, de pertinence et de pluralisme. Son objectif est de livrer à ce public les clés d'explication du fonctionnement du système socio-politique belge et de mettre en évidence les structures réelles du pouvoir, en Belgique et dans le cadre de l'Union européenne.

Le Courrier hebdomadaire paraît au rythme de 40 numéros par an, certaines livraisons correspondant à deux numéros. Chaque livraison est une monographie consacrée à l'étude approfondie d'un aspect de la vie politique, économique ou sociale au sens large. La revue du CRISP constitue depuis 1959 une source d'information incontournable sur des sujets variés : partis politiques, organisations représentatives d'intérêts sociaux et groupes de pression divers, évolution et fonctionnement des institutions, négociations communautaires, histoire politique, groupes d'entreprises et structures du tissu économique, conflits sociaux, enseignement, immigration, vie associative et culturelle, questions environnementales, européennes, etc. C'est également dans le Courrier hebdomadaire que sont publiés les résultats des élections commentés par le CRISP.

Les auteurs publiés sont soit des chercheurs du CRISP, formés en diverses disciplines des sciences humaines, soit des spécialistes extérieurs provenant des mondes scientifique, associatif et socio-politique. Dans tous les cas, les textes sont revus avant publication par le rédacteur en chef et par un groupe d'experts sélectionnés en fonction de la problématique abordée, afin de garantir la fiabilité de l'information proposée. Cette fiabilité, ainsi que la rigoureuse objectivité du Courrier hebdomadaire, constituent les atouts principaux d'une revue dont la qualité est établie et reconnue depuis près de 60 ans.

Fondateur : Jules Gérard-Libois

Président : Vincent de Coorebyter

Équipe de recherche :

Pierre Blaise (secrétaire général), Fabienne Collard, Vaïa Demertzis, Jean Faniel (directeur général), Christophe Goethals, Cédric Istasse, John Pitseys, David Van Den Abbeel (coordinateur du secteur Économie), Marcus Wunderle

Conseil d'administration :

Louise-Marie Bataille, Jacques Brassinne de La Buissière (vice-président honoraire), Vincent de Coorebyter (président), Francis Delpérée, Hugues Dumont, Éric Geerkens, Nadine Gouzée, Serge Govaert, Laura Iker, Patrick Lefevre, Michel Molitor (vice-président), Solveig Pahud, Pierre Reman (administrateur délégué), Robert Tollet (vice-président), Els Witte, Paul Wynants 


\section{Derniers numéros du Courrier hebdomadaire parus}

2361-2362 Le dossier Arco Serge Govaert

2359-2360 Évolution de la pratique de la Commission bancaire (1935-1975) Thibaud Giddey

2357-2358 Formation et spécificités historiques de la Commission bancaire (1935-1975) Thibaud Giddey

2355-2356 La Ligue des familles (1950-1975) Élodie Tallier

2353-2354 Le Processus de Kimberley et la lutte contre le commerce des « diamants de sang» Élise Rousseau

2352 Le projet de Centre de formation pour sportifs de haut niveau (CFSHN) Jérôme Defosse

2350-2351 L'autonomie constitutive des entités fédérées Quentin Peiffer

2348-2349 La régulation publique de la biomédecine. Procréation médicalement assistée, recherche sur embryons, gestation pour autrui Nathalie Schiffino

2346-2347 Distribution et redistribution des revenus : évolution des inégalités en Belgique Christian Valenduc

2344-2345 Le G1000 : une expérience citoyenne de démocratie délibérative M. Reuchamps, D. Caluwaerts, J. Dodeigne, V. Jacquet, J. Moskovic et S. Devillers

2343 Le profil des parlementaires néerlandophones en 2015 Jef Smulders

2341-2342 Grèves et conflictualité sociale en 2016 Iannis Gracos

La collection intégrale du Courrier hebdomadaire est accessible sur www.cairn.info.

L'accès est gratuit pour les numéros parus avant 2016.

Découvrez notre catalogue complet incluant nos autres publications sur www.crisp.be.

Pour être informé de nos publications dès leur parution, inscrivez-vous en ligne à notre lettre d'information électronique. 